\title{
FIELD EVALUATION OF RHIZOBACTERIAL INOCULANTS IN COMBINATION WITH HUMIC SUBSTANCES TO IMPROVE SEED AND OIL YIELDS OF SAFFLOWER (CARTHAMUS TINCTORIUS L.) UNDER IRRIGATED AND RAINFED CONDITIONS
}

\author{
EKIN, Z. \\ Department of Field Crops, Faculty of Agriculture, University of Van Yuzuncu Yil, Van, Turkey \\ (e-mail: zehraekin@yyu.edu.tr; phone: +90-432-225-1056; fax: phone: +90-432-225-1104) \\ (Received 24 $4^{\text {th }}$ Mar 2020; accepted $20^{\text {th }}$ Aug 2020)
}

\begin{abstract}
The present study was evaluated the application of humic acid (HA), which possess multifaceted biological action, and plant growth promoting rhizobacteria (PGPR) inoculation to improve the morphologic performance, yield and seed quality of safflower under different water regimes in the Eastern Anatolia region of Turkey. In a 2-year field trial, the two humic acid level (200 and $\left.400 \mathrm{~kg} \mathrm{ha}^{-1}\right)$ and the two rhizobacterial strains (Bacillus megatorium M3 and Bacillus subtilis OSU142) in together with control treatments were evaluated under irrigated and rainfed conditions using randomized complete block design with a split-split plot arrangement. Bacterial strains and humic acid levels highly increased plant growth, seed and oil yields, and seed nutrient contents under irrigated and rainfed conditions. However, the combined application of the OSU142 strain with $400 \mathrm{~kg} \mathrm{HA} \mathrm{ha-}{ }^{1}$ resulted in the greatest enhancement of safflower growth and yield under irrigated conditions, and seed and oil yields increased by 101.8 and $127.6 \%$ with treatment of $400 \mathrm{HA}+$ OSU14 respectively. Moreover, the integrated use of humic acids and PGPR inoculation relatively showed additive effect in the growth and yields of safflower under rainfed conditions, and increased by 186.5 with treatment of $400 \mathrm{HA}+$ OSU142 and $257.1 \%$ with treatment of $400 \mathrm{HA}+\mathrm{M}$ respectively.
\end{abstract}

Keywords: safflower, PGPR, humic acids, energy crop, agricultural sustainability, crop performance

\section{Introduction}

Safflower (Carthamus tinctorius L.) is a dryland oilseed crop with a very long cultivation history. It is widely grown for food, medicinal, cosmetic and biofuel purposes over a wide range of geographical areas from Far East to American continent (Ekin, 2005). Nowadays, scientific interest in this species has gained importance as a result of mainly their utilization increase in human nutrition and non-food production due to their high-quality vegetable oil. In safflower oil, the major fatty acids are linoleic and oleic acids and their relative proportions determine the safflower oil's functional properties and nutritional value (Cao et al., 2013; Rapson et al., 2015). On the basis of the nutritional value of safflower oil, oil quality, in fact, shows similarity to that of olive oil and is valued for human health reasons since the high unsaturated fatty acids (linoleic and oleic acids) contents leads to significant reduction in blood cholesterol levels (Dajue and Mündel, 1996; La Bella et al., 2019). In this regard, safflower would seem to be more favorable when compared to those of the oils of major oilseed crops such as sunflower, rapeseed, maize and soy commonly used for food and non-food sector in the semi-arid and marginal croplands of the world and central-eastern Turkey (Ekin, 2005; Kizil et al., 2008; Ozturk et al., 2008). In general, oilseed crops for application in the non-food sector, especially regarding biofuel research and development projects, have attract intensive attention in recent years in Turkey. In this regard, safflower appears to be more suitable as a dryland oilseed crop that can adapt 
highly to chancing climate and soil conditions for the agriculture and biofuels industry among other widely cultivated crops (Nosheen et al., 2018). However, it is characterized by spiny nature and low seed yield which have devitalized farmers from taking on its cultivation in the many countries including Turkey in dryland farming (Ekin, 2005; La Bella et al., 2019).

To maintain high yielding potential in safflower cultivation is firstly required water stress and soil fertility management in semi-arid and highland regions typically characterized by variable and unpredictable inadequate rainfall, poor moisture storing capacity of soils, large diurnal ranges in temperature and frequent strong winds (Ozturk et al., 2008). Due to readily deterioration of soil, water and other environmental resources in those regions compared to agriculture areas in different regions of the world, future food and energy demand and its created pressures are likely to further exacerbate the damaged soil health and effects of drought (Somerville and Briscoe, 2001). Therefore, it is imperative to protect soil health and productivity and to enhance the water stress tolerance of crops under the changing environmental conditions. In particular, drought tolerance will likely become increasingly significant for producing steady yields in all oilseed crops under global climate change (Comas et al., 2013). Although the safflower is well adapted to dryland cropping system, its agronomic performance and seed quality is particularly dependent upon climatic factors such as temperature and rainfall in flowering and seed maturation stages, and is largely affected by water stress conditions in these periods (Weiss, 1983; Kumar et al., 2016; La Bella et al., 2019). There are yet no economically current technological vehicles to facilitate plant production under drought. But the introduction of novel techniques to increase water stress tolerance is crucial for increasing safflower oil-based food and biofuel production. In this context, the role of beneficial rhizosphere microorganisms is gaining premium importance in the development of climate change resilient agriculture and stress management.

The rhizosphere of plants, the interface between plant roots and soil, is widely colonized by soil microorganisms (e.g. plant growth promoting rhizobacteria, PGPR). After inoculated on seed, PGPR can successfully colonize plant roots and improve plant growth directly by either releasing of plant growth stimulating compounds (e.g. phytohormones such as cytokines or auxins) or improving in nutrient uptakes (e.g. $\mathrm{N}_{2}$ fixing, P-solubilizing or siderophore release increasing nitrogen, phosphorus and iron availability, respectively) or indirectly by decreasing the harmful effects of pathogens via synthesis of antibiotics. Thus, the plant-bacteria symbioses could stimulate plant growth and yield by reducing dependency on traditional fertilizers, enhance the plant tolerance to both biotic and abiotic stresses, and serve to keep soil productivity and environmental health. However, the density and structure of the rhizosphere bacteria in the soil are dictated by soil $\mathrm{pH}$, organic matter content, water and nutrient availability throughout the root surface (Bossio et al., 1998; Drenovsky et al., 2004; Garcia-Pausas and Paterson, 2011; Backer et al., 2018). On the other hand, biogeographical patterns including topographic and climatic variations have also the powerful impacts on density of bacterial community (Kristin and Miranda, 2013). In the world, several PGPR species (Azotobacter, Azosprillum, Bacillus, Serratia, Enterobacter, Pseudomonas, Klebsiella and Variovorax) as rhizosphere-colonizing microorganisms were the subject of intense study in the field or laboratory in various areas of the many countries in recent years. In this context, it was generated valuable information on the various field crops, such as sugar beet and barley (Cakmakci et al., 200 2006), sugarcane (Silva et al., 
2017), sunflower (Shadid et al., 2012), wheat (Rosas et al., 2009; Hungria et al., 2010; Rana et al., 2012; Chandra et al., 2019), rice (Lucas et al., 2009), bean (Hoyos-Carvajal et al., 2009), canola (El-Howeity and Asfour, 2012), maize (Thonar et al., 2017) and soy (Cassán et al., 2009). However, each plant's responses to rhizobacteria cannot be expressed by admitted valid comprehension and this actually presents both an opportunity and a challenge. The capability of soil bacteria to reveal favorable effects on plant growth can be impaired under field conditions since other exterior factors come into play (Nelson, 2004; Backer et al., 2018; Chandra et al., 2019). Therefore, it has recently been demonstrated that the integrated use of humic substances and PGPR in agricultural practices could be envisaged as environmental-friendly technology to promote crop yield and quality. In addition, this sustainable agricultural approach can be a good means to enhance vegetable oil and biofuel production in water-deficit regions.

Humic substances, consisting of soil organic matter decomposition, are of crucial important for plant physiology and environmental protection by improving structure and fertility of soils and their resistance to erosion. However, they are a valuable complementary of soil microbiota, since they have natural hormone-like structure and exhibit biological activities. In addition, humic substances are show capacity to regulate the uptake and transport of nutrients to plants, improve crop yield and plant resistance to stress and effect root hair formation and lateral root development. Due to humic substances have a complex structure, which explains their versatile biological activity, the studies have majored on their interactions and structure with plants and associated rhizosphere microorganisms such as PGPR in controlled laboratory experiments. However, critical assessments of such interactions under real field conditions are necessary to quantify the current benefits on productivity, in view of improving practical modern technologies for advanced and sustainable agricultural systems purposed in enhancing crop yield and quality. Some authors (Canellas et al., 2013; Esringu et al., 2016; Schoebitz et al., 2016; Silva et al., 2017) suggested that coapplication of humic substances and PGPR inoculation in several crop species grown under field conditions can increase significantly macro- and micro nutrient uptakes and root growth, which in turn led to the increase crop yield. Moreover, these authors reported that stimulation of biological activity by humic acid can further enhance nutrient cycling through the action of microorganisms. Another reason given for increased crop yield, was also attributed to be highly adaptation of plant to several stress conditions by integrated use of humic substance and PGPR (Nardi et al., 2009; Dobbss et al., 2010; Busato et al., 2012; Puglisi et al., 2013; Canellas and Olivares, 2014; Olivares et al., 2015). Previous studies also reported that inoculation of specific plant growth promoting bacteria species like $\mathrm{N}_{2}$-fixing Bacillus subtilis and phosphorus solubilizing Bacillus megatorium instead of chemical fertilizers can serve as an environmental friendly alternative application and improve plant nutrition by increasing $\mathrm{N}$ and $\mathrm{P}$ uptake by plants (Cakmakci et al., 2001; Esitken et al., 2003). But, up till now no data are present in respect of the use of this Bacillus strains in safflower plants. However, only limited data are available with respect to the use of Pseudomonas, Azosprillum and Azotobacter spp., which were used to increase nitrogen uptake (Mirzakhani et al., 2009; Soleymanifard and Sidat, 2011; Sharifi et al., 2017; Nosheen et al., 2018), root morphology (Nosheen et al., 2011), and protein quantity and quality of seeds (Nosheen et al., 2016) in safflower. 
Safflower is an important oilseed plant that grows optimally in dry conditions, but is negatively affected by drought in the phenological periods (i.e. germination, stem elongation and branching, flowering), which are the most critical periods in terms of plant moisture requirement (Dajue and Mündel, 1996; Ekin, 2005). Thus, the combined use of humic acids and PGPR inoculation was explored as a possibility for improving agronomic performance of the safflower under irrigated and rainfed conditions in arid and semi-arid regions.

\section{Materials and methods}

\section{Site characterization}

The safflower (Carthamus tinctorius L.) variety Remzibey-05 was investigated for its response to plant growth promoting rhizobacteria inoculation and humic acid application under irrigated and rainfed field conditions. The field experiment was carried out at the research farm located in Ahlat district $\left(38^{\circ} 46^{\prime} \mathrm{N}\right.$ and $42^{\circ} 30^{\prime} \mathrm{E}$ with an altitude of $1722 \mathrm{~m}$ ) in the Eastern Anatolia region of Turkey during the cropping seasons 2010 and 2011. The climate of experimental area is continental with $562.6 \mathrm{~mm}$ total rainfall in long-term average (1958-2017), concentrated in winter. Annual mean air temperature and relative humidity values are $9.3{ }^{\circ} \mathrm{C}$ and $63.8 \%$, respectively. Maximum temperature is $22.8{ }^{\circ} \mathrm{C}$ in August and minimum temperature is $-2.5{ }^{\circ} \mathrm{C}$ in January. The weather data were collected from weather station close to the fields (Table 1). In 2010 and 2011 years, mean temperature values were $16.5{ }^{\circ} \mathrm{C}$ and $16.0{ }^{\circ} \mathrm{C}$ and amounts of rainfall were $291.0 \mathrm{~mm}$ and $269.4 \mathrm{~mm}$, respectively. The soil of experiment site was a silt-clay-loam, including $1.60 \%$ organic matter and $\mathrm{pH} 7$. total $\mathrm{N} 0.15 \mathrm{~g} / \mathrm{kg}, 6.8 \% \mathrm{CaCO}$ $1.16 \mathrm{dS} / \mathrm{m}$ electrical conductivity (EC), $0.15 \mathrm{~g} \mathrm{~kg}^{-1}$ total $\mathrm{N}, 7.95 \mathrm{mg} \mathrm{kg}^{-1}$ available $\mathrm{P}$, $3.30 \mathrm{mg} \mathrm{kg}^{-1}$ available $\mathrm{Mn}, 196 \mathrm{mg} \mathrm{kg}^{-1}$ available $\mathrm{K}, 5.85 \mathrm{mg} \mathrm{kg}^{-1}$ available $\mathrm{Fe}, 1.44 \mathrm{mg}$ $\mathrm{kg}^{-1}$ available $\mathrm{Zn}$ and $0.59 \mathrm{mg} \mathrm{kg}^{-1}$ available $\mathrm{Cu}$.

\section{Plant material and experimental design}

Safflower seeds were obtained from the Transitional Zone Agricultural Research Institute, Republic of Turkey Ministry of Agriculture and Forestry. The Remzibey-05 is an early-maturing safflower variety with Turkey of origin, drought-tolerant, spiny, open-pollinated, yellow flower color, white seed color, 32-35\% oil content, high-oleic oil type and approximately $1.00 \mathrm{t} \mathrm{ha}^{-1}$ dryland seed yield. The field experiments lay out was split-split plot design in randomized complete block with three replicates, with water regimes (irrigated and rainfed) as the main plot, humic acid (Control: without the addition of humic acid, $200 \mathrm{~kg} \mathrm{ha}^{-1}$ and $400 \mathrm{~kg} \mathrm{ha}^{-1}$ ) as the sub plots, and PGPR strains (Uninoculated control, Bacillus megatorium M3 and Bacillus subtilis OSU-142) as the sub-subplots. The humic acid doses were prepared by Agro-Lig ${ }^{\mathrm{TM}}$ (commercial products $-75 \%$ total organic matter, $65 \%$ total humic acid derived from leonardite, $22 \%$ max moisture, $\mathrm{pH} 3.5-5.5$ ) in granule form.

\section{Bacterial strains, culture media and seed bacterization}

Gram-positive, non-pathogenic Bacillus subtilis OSU-142 and Bacillus megatorium M3 strains were kindly obtained from Atatürk University-Turkey. In the previous studies, strains used in this study were determined that they showed capacity to grow in $\mathrm{N}$-free conditions, for hormones (IAA, GA 3 ), 1-aminocyclopropane-1carboxylate (ACC) 
deaminase and siderophore production, and to solubilize phosphate and to $\mathrm{N}_{2}$-fixing (Cakmakci et al., 2001; Orhan et al., 2006). For the inoculation of safflower seeds, bacterial cultures were grown on nutrient agar for routine use. Subsequently, single colony was transferred to $500 \mathrm{~mL}$ flasks containing nutrient broth. The cultures were incubated on a rotating shaker $(150 \mathrm{rpm})$ overnight at $28{ }^{\circ} \mathrm{C}$ aerobically, and then bacterial suspensions were diluted to final concentration at cell density of $1 \times 10^{9}$ colony forming unit $\mathrm{ml}^{-1}$ by sterilized and distilled water $\left(\mathrm{sdH}_{2} \mathrm{O}\right)$. Safflower seed surface was disinfected by dipping first in 3\% (v/v) sodium hypochlorite for $3 \mathrm{~min}$ and then $95 \%$ ethanol for $1 \mathrm{~min}$. After surface disinfection, the seeds were washed 3-4 times with $\mathrm{sdH}_{2} \mathrm{O}$. Approximately, $5 \mathrm{~g}$ sugar $\left(50 \mathrm{mg} \mathrm{mL}^{-1}\right)$ was added to each Erlenmeyer flasks, and the surface-sterilized seeds were soaked separately in this suspension. The seeds were treated with the bacteria in the flasks by shaking at $80 \mathrm{rpm}$ for $2 \mathrm{~h}$. For the uninoculated control, seeds were treated with sterile nutrient broth supplemented with sugar. After shaking, the seeds were taken out and air-dried on sterile Whatman filter paper sheets overnight.

Table 1. Mean, maximum and minimum temperature $\left({ }^{\circ} \mathrm{C}\right)$, monthly rainfall $(\mathrm{mm})$ and relative humidity (\%) trends in the experimental site (Ahlat, Turkey) during 2012011 and long-term average (LTA: 1958-2017)

\begin{tabular}{|c|c|c|c|c|c|c|}
\hline Year & Month & $\mathbf{T}_{\text {mean }}\left({ }^{\circ} \mathbf{C}\right)$ & $\mathbf{T}_{\max }\left({ }^{\circ} \mathbf{C}\right)$ & $\mathbf{T}_{\min }\left({ }^{\circ} \mathbf{C}\right)$ & Rainfall (mm) & R. Humidity \\
\hline \multirow{5}{*}{2010} & April & 7.3 & 14.5 & 3.4 & 154.4 & 71.0 \\
\hline & May & 11.4 & 16.9 & 7.3 & 106.2 & 65.8 \\
\hline & June & 18.3 & 24.7 & 11.9 & 28.0 & 50.4 \\
\hline & July & 22.8 & 29.3 & 16.1 & 1.8 & 37.3 \\
\hline & August & 22.5 & 29.2 & 15.7 & 0.6 & 35.6 \\
\hline \multirow{2}{*}{\multicolumn{2}{|c|}{$\begin{array}{c}\text { Season }(\mathbf{M} / \mathbf{T})^{*} \\
\text { Yearly }(\mathbf{M} / \mathbf{T})\end{array}$}} & 16.5 & 22.9 & 10.8 & 291.0 & 52.0 \\
\hline & & 10.9 & 16.6 & 5.2 & 399.0 & 59.6 \\
\hline \multirow{5}{*}{2011} & April & 6.9 & 11.0 & 3.3 & 159.0 & 71.0 \\
\hline & May & 11.2 & 16.6 & 6.7 & 90.0 & 69.1 \\
\hline & June & 17.6 & 23.6 & 11.5 & 15.6 & 52.1 \\
\hline & July & 22.3 & 28.9 & 15.8 & 3.2 & 41.3 \\
\hline & August & 22.0 & 28.4 & 15.4 & 1.6 & 40.4 \\
\hline \multicolumn{2}{|c|}{ Season $(M / T)$} & 16.0 & 21.7 & 10.5 & 269.4 & 54.8 \\
\hline \multicolumn{2}{|c|}{ Yearly (M/T) } & 8.6 & 13.7 & 4.0 & 566.6 & 56.4 \\
\hline \multirow{5}{*}{ LTA } & April & 6.9 & 11.4 & 2.8 & 87.1 & 69.4 \\
\hline & May & 13.1 & 17.0 & 7.1 & 70.2 & 65.0 \\
\hline & June & 18.9 & 23.3 & 11.2 & 28.7 & 55.6 \\
\hline & July & 21.5 & 28.0 & 15.3 & 8.3 & 49.4 \\
\hline & August & 22.8 & 28.3 & 15.4 & 5.7 & 47.7 \\
\hline \multirow{2}{*}{\multicolumn{2}{|c|}{$\begin{array}{l}\text { Season }(M / T) \\
\text { Yearly }(M / T)\end{array}$}} & 16.1 & 21.6 & 10.4 & 136.2 & 57.4 \\
\hline & & 9.3 & 14.2 & 4.5 & 562.6 & 63.8 \\
\hline
\end{tabular}

* M: Mean, T: Total 


\section{Main cultivation practices and measurement of morphologic and yield data}

The field experiment was carried out under rainfed and irrigated conditions. After soil was ploughed and harrowed, safflower seeds were sown at $40 \mathrm{~cm}$ row spacing on early April in both years. Overall, plant density was 40 viable seeds $\mathrm{m}^{-2}$ and sub-subplots area was $16 \mathrm{~m}^{2}$. At the start of the field experiment, a basal dose of $\mathrm{N}$ (urea) and $\mathrm{P}$ (Triple Super Phosphate), at rates of 300 and $150 \mathrm{~kg}^{-}$which might support root morphology and plant growth improvements in safflower (Nosheen et al., 2011), were applied to the plants. Humic acids were applied in the soil according to layout at sowing time and then mixed well in the soil. In the study, irrigation was applied based on the phenological periods (stem elongation and flowering), which are the most critical periods in terms of plant moisture requirement in safflower. This application called as supplemental irrigation, as a notion, refers to providing significant increases in yield by applying irrigation water in support of rainfall in plants grown under rainfall conditions as the most accurate approach in irrigation water management in the arid and semi-arid regions. In the study, the soil moisture has been allowed to fluctuate only at field capacity range, so soil water potential was controlled in the range between -10 and $-20 \mathrm{kPa}$ as indicated by Tensiometer at a depth of $15 \mathrm{~cm}$ below the soil surface in the irrigated treatment. Water was applied uniformly across all irrigated plots. Each of irrigations applied in the stem elongation (June) and flowering (July) growth periods represented a total of approximately $48 \mathrm{~mm}$ and $80 \mathrm{~mm}$ in 201 and 56 and $85 \mathrm{~mm}$ rainfall equivalent, respectively. Therefore, the total quantity of water received in irrigation conditions was $419.0 \mathrm{~mm}$ and $410.4 \mathrm{~mm}$ in the five-month cycle in 2010 and 2011 years, respectively. Weeds were controlled by hand. Safflower plants were harvested at the physiological maturity in mid-August in both years. Seed moisture content was also below 8.00\%.

Morphologic and yield characterization were performed following the guide of International Plant Genetic Resources Institute for safflower. Each treatment was evaluated for 5 pre-harvest and 17 post-harvest traits. The pre-harvest traits were stem diameter $(\mathrm{mm})$, plant height $(\mathrm{cm})$, number (no.) of primary branches per plant, capitilum diameter (cm) and number (no.) of capitula per plant. Data was recorded for twenty healthy plants in each of 54 experimental plots. The post-harvest traits were number (no.) of seeds per capitulum, fertile and sterile seed percentage $(\%), 1000$-seed weight $(\mathrm{g})$, seed oil and protein contents $(\%)$, seed, oil and protein yields $\left(\mathrm{t} \mathrm{ha}^{-1}\right)$ and mineral composition of safflower seeds $(\mathrm{mg} / \mathrm{kg})$. Plant height was measured as main shoot length from soil surface to the uppermost capitulum of the plant. Diameters of stem and capitilum were measured by digital caliper. The total number of capitula and branches was recorded as number of capitilum and primary branches per plant. Thousand seed weight was measured as seed weight of 1000 achenes from each of plants in grams. Seed yield was recorded at the harvest area of $7.2 \mathrm{~m}^{2}$ after removing the two rows border plants all around. Seed oil, protein and nutrient contents were analyzed in three replicates. Oil content was measured by Soxhalet extraction method using hexane as solvent. Protein content in seed samples was calculated from nitrogen using the conversional factor of 6.25 and determined by the Kjeldahl method for nitrogen content measurement in safflower. Magnesium $(\mathrm{Mg})$, potassium $(\mathrm{K})$, calcium $(\mathrm{Ca})$, phosphorus $(\mathrm{P})$, zinc $(\mathrm{Zn})$, manganese $(\mathrm{Mn})$, copper $(\mathrm{Cu})$ and iron $(\mathrm{Fe})$ contents in seed samples were determined by wet-digesting the ground seeds using $\mathrm{HNO}_{3}: \mathrm{HClO}_{4}(6: 2 \mathrm{v} / \mathrm{v})$ by Advanced Microwave Digestion System. Subsequently, mineral nutrients composition was evaluated by Inductively Coupled Plasma Optical Emission Spectrometry (iCAP 6000 SERIES, ICP Spectrometer) as per manufacturer's instructions. 


\section{Statistical analysis}

In order to reveal if data are suitable for normal distribution and homogeneity, the Kolmogorov-Smirnov normality and Bartlett homogeneity tests were used. Afterwards, analysis of variance (ANOVA) was used to compare the effects of water regimes, humic acids and PGPR treatments interactions for 2 years using PROC GLM of SAS 9. Mean comparisons were conducted using Duncan multiple comparison test (DMRT). All the experiments were performed in three replicates and data were presented as descriptive statistics (means; $\mathrm{n}=3$ ). Since there were no significant year $\mathrm{x}$ treatment interactions for the tested most traits $(\mathrm{p}<0.05), 2$-year data were combined and used for the mean comparisons.

\section{Results}

\section{Analysis of pre-harvest morphologic traits}

The analysis of combined ANOVA showed significant differences by the tested factors (humic acids and PGPR inoculation) under both irrigated and rainfed conditions, as pointed by variations in multiple biometric characteristics of safflower growth (Table 2). Results from analysis of pre-harvest morphologic traits indicated that plant height significantly differed between soil moisture conditions. On average, the highest plant height was recorded in irrigated conditions. Plant height significantly $(\mathrm{P}<0.01)$ increased with increasing doses of humic acid. Likewise, inoculations of OSU142 and M3 significantly $(\mathrm{P}<0.01)$ increased plant height by 26.20 and $15.57 \%$, over that of the uninoculated control, respectively. Significantly $(\mathrm{P}<0.01)$ interactions were observed for $\mathrm{W} \times \mathrm{HA}, \mathrm{W} \times \mathrm{P}, \mathrm{HA} \times \mathrm{P}$ and $\mathrm{W} \times \mathrm{HA} \times \mathrm{P}$ (Table 2).

The mean values for the $\mathrm{W} \times \mathrm{P}$ and $\mathrm{W} \times \mathrm{HA}$ interactions (Table 3) revealed that, for both irrigated and rainfed conditions, the OSU142 strain and the $400 \mathrm{~kg} \mathrm{HA} \mathrm{ha}^{-1}$ treatment had the highest values for plant height, over their respective control treatments. Similarly, the interaction of HA x P (Table 4) indicated that humic acid and rhizobacterial inoculation increased the plant height, with $400 \mathrm{~kg} \mathrm{HA} \mathrm{ha}^{-1}$ and the OSU142 strain being the most effective. Moreover, there was significant $(\mathrm{P}<0.01) \mathrm{W} x$ HA x P interaction (Fig. 1) for plant height, and the highest plant height was recorded for plants treated with $400 \mathrm{HA}+$ OSU142 in irrigated condition maintaining an increase of $47.8 \%$, compared to their respective non-HA uninoculated control.

Significant differences $(\mathrm{P}<0.01)$ in the stem diameter and number of primary branches per plant of safflower were observed for water regimes, HA, and PGPR inoculation (Table 2). Irrigation and HA applications considerably increased the both stem diameter and number of primary branches per plant of safflower, with the highest values noted for the $400 \mathrm{HA} \mathrm{ha}^{-1}$ treatment. PGPR inoculation also significantly increased stem diameter and number of primary branches, with higher values noted for OSU142 (Bacillus subtilis) than for M3 (Bacillus megatorium) and the uninoculated control. In addition, there were significant $(\mathrm{P}<0.01) \mathrm{W} \times \mathrm{P}$ and HA x P interactions for the stem diameter of safflower (Table 2). Mean values for the $\mathrm{W} \times \mathrm{P}$ interaction (Table 3) suggested that the highest stem diameter $(7.97 \mathrm{~mm})$ was for the OSU142 strain under irrigated conditions maintaining an increase of $22.7 \%$, over their respective control treatment. The interaction of HA x P (Table 4) revealed that, for the 200 or 400 HA + OSU142 and the $400 \mathrm{HA}+\mathrm{M} 3$ treatments had the highest values for stem diameter (7.5 7.39 and $7.50 \mathrm{~mm}$, respectively). 
Table 2. Pre-harvest morphological traits of safflower affected by humic acid application and bacterial inoculation under different water regimes (means of the combined years) ${ }^{l}$

\begin{tabular}{|c|c|c|c|c|c|c|}
\hline & & \begin{tabular}{|c|}
$\begin{array}{c}\text { Plant height } \\
(\mathrm{cm})\end{array}$ \\
\end{tabular} & $\begin{array}{c}\text { Stem diameter } \\
(\mathrm{mm})\end{array}$ & \begin{tabular}{|c|} 
No. of \\
branches/plant
\end{tabular} & $\begin{array}{c}\text { No. of } \\
\text { capitula/plant }\end{array}$ & $\begin{array}{c}\text { Capitilum } \\
\text { diameter }(\mathbf{c m})\end{array}$ \\
\hline \multicolumn{7}{|c|}{ WATER REGIMES $(\mathbf{W})$} \\
\hline \multicolumn{2}{|l|}{ Irrigated } & $73.8 \mathrm{a}$ & $7.39 \mathrm{a}$ & $9.5 \mathrm{a}$ & $11.6 \mathrm{a}$ & $2.51 \mathrm{a}$ \\
\hline \multicolumn{2}{|l|}{ Rainfed } & $59.5 \mathrm{~b}$ & $5.64 \mathrm{~b}$ & $5.9 \mathrm{~b}$ & $8.5 \mathrm{~b}$ & $2.28 \mathrm{~b}$ \\
\hline \multicolumn{7}{|c|}{ HUMIC ACID (HA) } \\
\hline \multicolumn{2}{|l|}{0} & $59.7 \mathrm{c}$ & $5.97 \mathrm{c}$ & $6.9 \mathrm{c}$ & $7.8 \mathrm{c}$ & $2.24 \mathrm{c}$ \\
\hline \multicolumn{2}{|l|}{200} & $68.2 \mathrm{~b}$ & $6.58 \mathrm{~b}$ & $7.8 \mathrm{~b}$ & $10.6 \mathrm{~b}$ & $2.42 \mathrm{~b}$ \\
\hline \multicolumn{2}{|l|}{400} & $72.0 \mathrm{a}$ & $7.00 \mathrm{a}$ & $8.5 \mathrm{a}$ & $11.8 \mathrm{a}$ & $2.53 \mathrm{a}$ \\
\hline \multicolumn{7}{|c|}{ PGPR STRAINS (P) } \\
\hline \multicolumn{2}{|c|}{ Uninoculated } & $58.0 \mathrm{c}$ & $5.78 \mathrm{c}$ & $6.8 \mathrm{c}$ & $8.6 \mathrm{c}$ & $2.29 \mathrm{c}$ \\
\hline \multicolumn{2}{|l|}{ OSU142 } & $73.2 \mathrm{a}$ & $7.15 \mathrm{a}$ & $8.5 \mathrm{a}$ & $11.5 \mathrm{a}$ & $2.51 \mathrm{a}$ \\
\hline \multicolumn{2}{|l|}{ M3 } & $68.7 \mathrm{~b}$ & $6.60 \mathrm{~b}$ & $7.7 \mathrm{~b}$ & $10.1 \mathrm{~b}$ & $2.40 \mathrm{~b}$ \\
\hline \multicolumn{7}{|c|}{ SIGNIFICANCE } \\
\hline $\begin{array}{l}\text { Source } \\
\end{array}$ & df & \multicolumn{5}{|c|}{ Mean square } \\
\hline Year $(Y)$ & 1 & $288.12 *$ & $1.13^{\mathrm{ns}}$ & $21.87^{*}$ & $32.34 * *$ & $0.0800^{\mathrm{ns}}$ \\
\hline W & 1 & $5532.67^{* *}$ & $82.51^{* *}$ & $347.76^{* *}$ & $251.16^{* *}$ & $1.52^{* *}$ \\
\hline Y x W & 1 & $28.83 *$ & $0.08^{\mathrm{ns}}$ & $0.71^{\mathrm{ns}}$ & $0.46^{\mathrm{ns}}$ & $0.0003^{\mathrm{ns}}$ \\
\hline HA & 2 & $1417.95^{* *}$ & $9.60^{* *}$ & $22.83^{* *}$ & $156.20^{* *}$ & $0.74^{* *}$ \\
\hline Y x HA & 2 & $16.83 *$ & $0.12^{\mathrm{ns}}$ & $0.22^{\mathrm{ns}}$ & $0.87^{\mathrm{ns}}$ & $0.0001^{\mathrm{ns}}$ \\
\hline $\mathrm{P}$ & 2 & $2199.35^{* *}$ & $17.24^{* *}$ & $23.93^{* *}$ & $75.69^{* *}$ & $0.41^{* *}$ \\
\hline $\mathrm{Y} \times \mathrm{P}$ & 2 & $6.39^{\mathrm{ns}}$ & $0.02^{\mathrm{ns}}$ & $0.19^{\text {ns }}$ & $0.84^{\mathrm{ns}}$ & $0.0016^{\mathrm{ns}}$ \\
\hline $\mathrm{W} \times \mathrm{HA}$ & 2 & $74.41^{* *}$ & $0.01^{\mathrm{ns}}$ & $0.11^{\mathrm{ns}}$ & $4.50^{* *}$ & $0.07^{* *}$ \\
\hline Y x W x HA & 2 & $19.68^{*}$ & $0.11^{\mathrm{ns}}$ & $0.28^{\mathrm{ns}}$ & $0.46^{\mathrm{ns}}$ & $0.0005^{\mathrm{ns}}$ \\
\hline W x P & 2 & $299.87^{* *}$ & $1.34^{* *}$ & $0.06^{\mathrm{ns}}$ & $4.69^{* *}$ & $0.02^{* *}$ \\
\hline $\mathrm{Y} \times \mathrm{W} \times \mathrm{P}$ & 2 & $0.27^{\mathrm{ns}}$ & $0.07^{\mathrm{ns}}$ & $0.17^{\mathrm{ns}}$ & $0.21^{\mathrm{ns}}$ & $0.0010^{\mathrm{ns}}$ \\
\hline $\mathrm{HA} \times \mathrm{P}$ & 4 & $62.72^{* *}$ & $1.49^{* *}$ & $0.25^{\mathrm{ns}}$ & $1.37^{* *}$ & $0.009^{*}$ \\
\hline Y x HA x P & 4 & $6.77^{\mathrm{ns}}$ & $0.16^{\mathrm{ns}}$ & $0.04^{\mathrm{ns}}$ & $0.14^{\mathrm{ns}}$ & $0.0013^{\mathrm{ns}}$ \\
\hline W x HA x P & 4 & $45.01^{* *}$ & $0.26^{\mathrm{ns}}$ & $0.47^{\mathrm{ns}}$ & $3.17^{* *}$ & $0.004^{\mathrm{ns}}$ \\
\hline $\mathrm{CV}(\%)$ & & 5.09 & 9.24 & 6.23 & 4.24 & 5.33 \\
\hline
\end{tabular}

${ }^{1}$ The means were obtained from combined analysis of variance. $\mathrm{ns},{ }^{*}$, and ${ }^{* *}$ represent nonsignificant and significant at $\mathrm{P} \leq 0.05$ and $\mathrm{P} \leq 0.01$ according to DMRT, respectively. $\mathrm{CV}$ : coefficient of variations

Number of capitula per plant and capitilum diameter for safflower were severely decreased by rainfed condition, whereas it increased with the application of HA and PGPR inoculation (Table 2). Furthermore, significant $(\mathrm{P}<0.01)$ interactions were observed for $\mathrm{W} \times \mathrm{HA}, \mathrm{W} \times \mathrm{P}$ and $\mathrm{HA} \times \mathrm{P}$ for these parameters. The $\mathrm{W} \times \mathrm{HA}$ and $\mathrm{W} \times \mathrm{P}$ interactions (Table 3) revealed that the highest number of capitula per plant and capitilum diameter for safflower was for the $400 \mathrm{~kg} \mathrm{HA} \mathrm{ha}^{-1}$ treatment and the OSU142 inoculation under the irrigated conditions, whereas the lowest values were observed for the without HA control and the uninoculated control under rainfed conditions. Likewise, the HA x P interaction showed that OSU142 significantly enhanced number of capitula per plant and capitilum diameter in the $400 \mathrm{~kg} \mathrm{HA} \mathrm{ha}^{-1}$ treatment by $104.6 \%$ and $22.2 \%$ over that of without HA and the uninoculated control, respectively. Mean values for the 
W x HA x P interaction (Fig. 2) suggested that the highest number of capitula per plant was recorded for plants treated with $400 \mathrm{HA}+$ OSU142 under irrigated conditions maintaining an increase of $63.6 \%$, whereas the plants submitted to rainfed condition showed considerably increase rate of $181.4 \%$ in number of capitula per plant in the 400 HA + OSU142 treatment, over their respective control treatments.

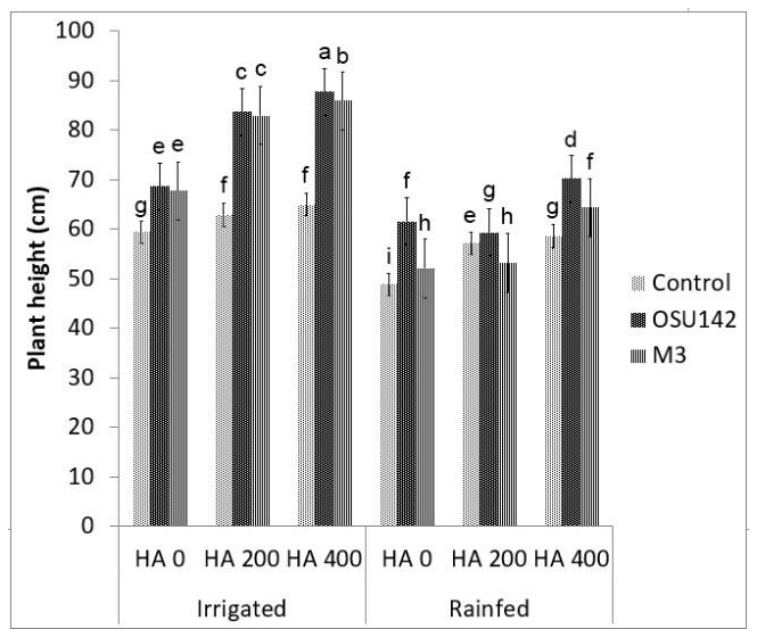

Figure 1. The interactive effect of water regimes, humic acids and PGPR on the plant height of safflower. Different letters along with water regimes, humic acids and PGPR treatments indicate significant differences in access treatment means from three replications tested at $P \leq 0.05$ according to DMRT

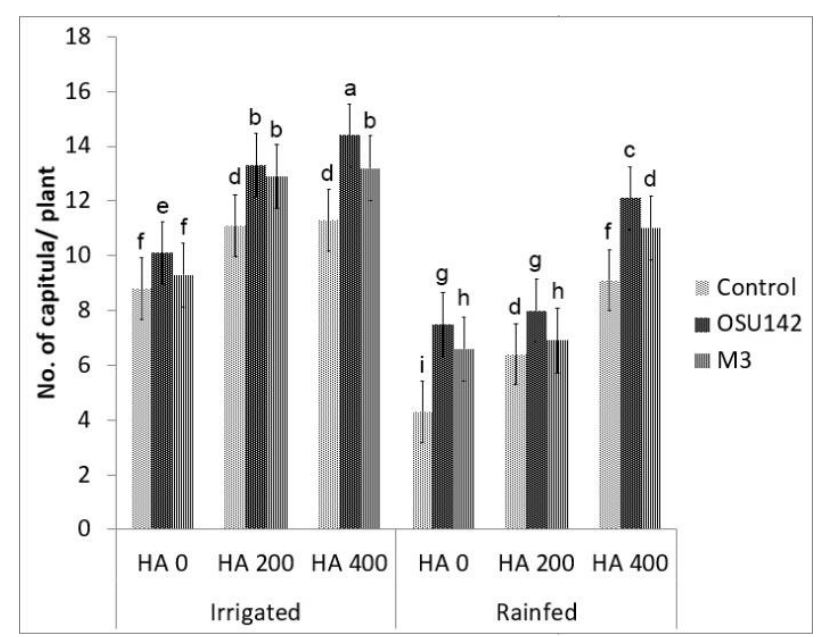

Figure 2. The interactive effect of water regimes, humic acids and PGPR on the number of capitula per plant of safflower. Different letters along with water regimes, humic acids and $P G P R$ treatments indicate significant differences in access treatment means from three replications tested at $P \leq 0.05$ according to DMRT

\section{Analysis of post-harvest seed traits}

The post-harvest seed traits of safflower (number of seeds per capitulum, thousand seed weight, and fertile and sterile seed percentage) were significantly $(\mathrm{P}<0.01)$ affected by all the treatments (Table 5). Irrigation application considerably increased the 
number of seeds per capitulum, thousand seed weight, and fertile seed percentage, whereas the highest sterile seed percentage was noted for the rainfed condition. HA and rhizobacterial inoculation increased all the seed traits of safflower, except for sterile seed percentage. Table 5 shows the significant $(\mathrm{P}<0.01)$ interactions of $\mathrm{W} \times \mathrm{HA}, \mathrm{W} \times$ $\mathrm{P}, \mathrm{HA} \times \mathrm{P}$ and $\mathrm{W} \times \mathrm{HA} \times \mathrm{P}$ for number of seeds per capitulum.

Table 3. Interaction between humic acid (HA) and water regimes (irrigated and rainfed);

$P G P R$ strain and water regimes on pre-harvest traits of safflower

\begin{tabular}{|c|c|c|c|c|c|c|c|c|c|c|c|c|c|c|c|c|}
\hline \multirow[t]{2}{*}{ Factors } & \multicolumn{2}{|c|}{$\begin{array}{c}\text { Plant height } \\
\text { (cm) }\end{array}$} & \multicolumn{2}{|c|}{$\begin{array}{c}\begin{array}{c}\text { Stem } \\
\text { diameter } \\
(\mathbf{m m})\end{array} \\
\end{array}$} & \multicolumn{2}{|c|}{$\begin{array}{l}\text { No. of } \\
\text { capitula/ } \\
\text { plant }\end{array}$} & \multicolumn{2}{|c|}{$\begin{array}{c}\text { Capitulum } \\
\text { diameter } \\
(\mathbf{c m})\end{array}$} & \multicolumn{2}{|c|}{$\begin{array}{c}\text { No. of seeds/ } \\
\text { capitulum }\end{array}$} & \multicolumn{2}{|c|}{$\begin{array}{l}1000 \text { seed } \\
\text { weight }(\mathrm{g})\end{array}$} & \multicolumn{2}{|c|}{$\begin{array}{l}\text { Seed yield } \\
\qquad\left(\mathrm{t} \mathrm{ha}^{-1}\right)\end{array}$} & \multicolumn{2}{|c|}{$\begin{array}{c}\text { Oil yield } \\
\left(t \text { ha }^{-1}\right)\end{array}$} \\
\hline & $\mathbf{I}$ & $\mathbf{R}$ & $\mathbf{I}$ & $\mathbf{R}$ & $\mathbf{I}$ & $\mathbf{R}$ & $\mathbf{I}$ & $\mathbf{R}$ & I & $\mathbf{R}$ & I & $\mathbf{R}$ & I & $\mathbf{R}$ & I & $\mathbf{R}$ \\
\hline \multicolumn{17}{|l|}{ HA $\left(\mathrm{kg} \mathrm{ha}^{-1}\right)$} \\
\hline 0 & $.3^{c}$ & $54.2^{\mathrm{e}}$ & 6.85 & 5.09 & $9.4^{\mathrm{d}}$ & $6.1^{\mathrm{f}}$ & $2.41^{\mathrm{c}}$ & $2.08^{\mathrm{e}}$ & $28.1^{\mathrm{c}}$ & $23.9^{\mathrm{d}}$ & $38.2^{b}$ & $36.3^{\mathrm{c}}$ & $1.89^{\mathrm{c}}$ & $1.02^{\mathrm{e}}$ & $0.61^{\mathrm{c}}$ & $0.29^{\mathrm{e}}$ \\
\hline 2 & ${ }^{\mathrm{b}}$ & $59.8^{\mathrm{d}}$ & 7 & 5.68 & $12.4^{\mathrm{b}}$ & $7.4^{\mathrm{e}}$ & $2.54^{\mathrm{b}}$ & $2.31^{\mathrm{d}}$ & $34.4^{\mathrm{a}}$ & $24.4^{\mathrm{d}}$ & $41.5^{\mathrm{a}}$ & $38.8^{b}$ & & $1.37^{\mathrm{d}}$ & $0.99^{\mathrm{b}}$ & \\
\hline 400 & $79.6^{\mathrm{a}}$ & $64.4^{\mathrm{c}}$ & 7.85 & 6.14 & $13.0^{\mathrm{a}}$ & $10.7^{\mathrm{c}}$ & $2.60^{\mathrm{a}}$ & $2.44^{\mathrm{c}}$ & $32.5^{\mathrm{b}}$ & $28.2^{\mathrm{c}}$ & $42.4^{\mathrm{a}}$ & $39.7^{\mathrm{b}}$ & $3.04^{\mathrm{a}}$ & $1.87^{\mathrm{c}}$ & $1.10^{\mathrm{a}}$ & $0.60^{\mathrm{c}}$ \\
\hline \multicolumn{17}{|c|}{ PGPR STRAIN } \\
\hline ninoculated & $62.4^{\mathrm{d}}$ & $53.6^{\mathrm{f}}$ & $6.51^{\mathrm{c}}$ & $5.06^{\mathrm{e}}$ & $10.4^{\mathrm{c}}$ & $6.8^{\mathrm{e}}$ & $2.43^{\mathrm{c}}$ & $2.15^{\mathrm{e}}$ & $29.6^{\mathrm{b}}$ & $24.0^{\mathrm{e}}$ & 39.2 & 36.8 & $2.09^{\mathrm{c}}$ & $1.15^{\mathrm{f}}$ & $0.69^{\mathrm{b}}$ & $0.33^{\mathrm{e}}$ \\
\hline OSU142 & $80.1^{\mathrm{a}}$ & $66.4^{\mathrm{c}}$ & $7.97^{\mathrm{a}}$ & $6.35^{\mathrm{c}}$ & $12.6^{\mathrm{a}}$ & $10.4^{\mathrm{c}}$ & $2.59^{\mathrm{a}}$ & $2.42^{\mathrm{c}}$ & $33.2^{\mathrm{a}}$ & $28.5^{\mathrm{c}}$ & 41.7 & 39.2 & $2.92^{\mathrm{a}}$ & $1.82^{\mathrm{d}}$ & $1.01^{\mathrm{a}}$ & $0.57^{\mathrm{c}}$ \\
\hline M3 & $78.9^{\mathrm{b}}$ & $58.4^{\mathrm{e}}$ & $7.70^{b}$ & $5.52^{\mathrm{d}}$ & $11.8^{\mathrm{b}}$ & $8.5^{d}$ & $2.52^{b}$ & $2.27^{\mathrm{d}}$ & $32.1^{\mathrm{a}}$ & $26.2^{d}$ & 41.3 & 38.8 & $2.75^{b}$ & $1.41^{\mathrm{e}}$ & $0.98^{\mathrm{a}}$ & $0.46^{\mathrm{d}}$ \\
\hline
\end{tabular}

Means having different letters are significantly different at $\mathrm{P} \leq 0.05$ according to DMRT

Table 4. Interaction between humic acid (HA) and PGPR strain on growth and yield of safflower

\begin{tabular}{|c|c|c|c|c|c|c|c|c|c|}
\hline $\begin{array}{c}\text { HA } \\
\left(\mathrm{kg} \mathrm{ha}^{-1}\right)\end{array}$ & PGPR strain & $\begin{array}{c}\text { Plant } \\
\text { height } \\
(\mathrm{cm})\end{array}$ & $\begin{array}{c}\text { Stem } \\
\text { diameter } \\
(\mathbf{m m})\end{array}$ & $\begin{array}{c}\text { No. of } \\
\text { capitula per } \\
\text { plant }\end{array}$ & $\begin{array}{l}\text { No. of seeds } \\
\text { per capitulum }\end{array}$ & $\begin{array}{l}\text { Capitilum } \\
\text { diameter } \\
(\mathrm{cm})\end{array}$ & 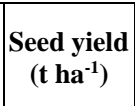 & $\begin{array}{c}\text { Oil yield } \\
\left(\mathbf{t} \mathbf{h a}^{-1}\right)\end{array}$ & $\begin{array}{c}\text { Protein } \\
\text { yield }\left(t \text { ha }^{-1}\right)\end{array}$ \\
\hline \multirow{3}{*}{0} & Uninoculated & $54.1 \mathrm{~h}$ & $5.37 \mathrm{~d}$ & $6.5 \mathrm{f}$ & $24.8 \mathrm{~b}$ & $2.16 \mathrm{f}$ & $1.20 \mathrm{f}$ & $0.35 \mathrm{f}$ & $0.15 \mathrm{f}$ \\
\hline & OSU142 & $65.2 \mathrm{~d}$ & $6.55 \mathrm{~b}$ & $8.8 \mathrm{~d}$ & $27.0 \mathrm{~b}$ & $2.32 \mathrm{~d}$ & $1.68 \mathrm{~d}$ & $0.52 \mathrm{e}$ & $0.23 \mathrm{~d}$ \\
\hline & M3 & $60.0 \mathrm{f}$ & $6.00 \mathrm{c}$ & $8.0 \mathrm{e}$ & $26.2 \mathrm{~b}$ & $2.25 \mathrm{e}$ & $1.48 \mathrm{e}$ & $0.49 \mathrm{e}$ & $0.19 \mathrm{e}$ \\
\hline \multirow{3}{*}{200} & Uninoculated & $58.1 \mathrm{~g}$ & $5.88 \mathrm{c}$ & $9.0 \mathrm{~d}$ & $26.8 \mathrm{~b}$ & $2.30 \mathrm{~d}$ & $1.69 \mathrm{~d}$ & $0.55 \mathrm{e}$ & $0.25 \mathrm{~d}$ \\
\hline & OSU142 & $75.4 \mathrm{~b}$ & $7.54 \mathrm{a}$ & $12.3 \mathrm{~b}$ & $33.2 \mathrm{a}$ & $2.56 \mathrm{~b}$ & $2.47 \mathrm{a}$ & $0.84 \mathrm{~b}$ & $0.35 \mathrm{~b}$ \\
\hline & M3 & $71.2 \mathrm{c}$ & $6.33 \mathrm{bc}$ & $10.4 \mathrm{c}$ & $31.7 \mathrm{a}$ & $2.41 \mathrm{c}$ & $2.03 \mathrm{c}$ & $0.71 \mathrm{c}$ & $0.27 \mathrm{c}$ \\
\hline \multirow{3}{*}{400} & Uninoculated & $61.8 \mathrm{e}$ & $6.11 \mathrm{c}$ & $10.2 \mathrm{c}$ & $27.2 \mathrm{~b}$ & $2.41 \mathrm{c}$ & $1.97 \mathrm{c}$ & $0.64 \mathrm{~d}$ & $0.29 \mathrm{c}$ \\
\hline & OSU142 & $79.0 \mathrm{a}$ & $7.39 \mathrm{a}$ & $13.3 \mathrm{a}$ & $30.5 \mathrm{a}$ & $2.64 \mathrm{a}$ & $2.64 \mathrm{a}$ & $0.92 \mathrm{a}$ & $0.39 \mathrm{a}$ \\
\hline & M3 & $75.2 \mathrm{~b}$ & $7.50 \mathrm{a}$ & $12.1 \mathrm{~b}$ & $31.3 \mathrm{a}$ & $2.53 \mathrm{~b}$ & $2.44 \mathrm{~b}$ & $0.87 \mathrm{~b}$ & $0.32 \mathrm{~b}$ \\
\hline
\end{tabular}

Means having different letters are significantly different at $\mathrm{P} \leq 0.05$ according to DMRT

The $\mathrm{W} \times \mathrm{HA}$ interaction (Table 3) revealed the highest number of seeds per capitulum for the $200 \mathrm{~kg} \mathrm{HA} \mathrm{ha} \mathrm{k}^{-1}$ treatment under irrigated condition, from that of the non-HA treatment. Conversely, it was also noted increased with increasing humic acid under rainfed condition. The $\mathrm{W} \times \mathrm{P}$ interactions means (Table 3) indicated the highest number of seeds per capitulum of safflower was noted for all the bacterial inoculations under irrigated conditions, whereas the plants inoculated with the OSU142 strain increased the number of seeds per capitulum under both irrigated and rainfed conditions by 12.2 and $18.8 \%$, respectively, over their respective control treatments. Similarly, HA $\mathrm{x} \mathrm{P}$ interactions means (Table 4) revealed that all the bacterial inoculations significantly increased the number of seeds per capitulum in all levels of HA treatment, from that of the control. In addition, mean values for the $\mathrm{W} \times \mathrm{HA} \times \mathrm{P}$ interaction (Fig. 3) revealed 
that the applications of $200 \mathrm{HA}+\mathrm{M} 3$ and $200 \mathrm{HA}+$ OSU142 under irrigated conditions resulted in the highest number of seeds per capitulum by increases of $41.4 \%$ and $36.5 \%$, whereas the plants submitted to rainfed condition showed considerably increase rates of $28.7 \%$ and $27.0 \%$ in number of capitula per plant in the $400 \mathrm{HA}+\mathrm{M} 3$ and 400 $\mathrm{HA}+$ OSU142 treatments, over their respective control treatments.

Table 5. Post-harvest seed traits of safflower affected by humic acid application and bacterial inoculation under different water regimes

\begin{tabular}{|c|c|c|c|c|c|}
\hline & & $\begin{array}{c}\text { No. of } \\
\text { seeds/capitulum }\end{array}$ & $\begin{array}{l}1000 \text { seed } \\
\text { weight }(\mathrm{g}) \\
\end{array}$ & $\begin{array}{c}\text { Fertile seed } \\
\text { percentage }(\%)\end{array}$ & $\begin{array}{c}\text { Sterile seed } \\
\text { percentage }(\%)\end{array}$ \\
\hline \multicolumn{6}{|c|}{ WATER REGIMES (W) } \\
\hline \multicolumn{2}{|l|}{ Irrigated } & $31.7 \mathrm{a}$ & $40.7 \mathrm{a}$ & $95.90 \mathrm{a}$ & $4.12 \mathrm{~b}$ \\
\hline \multicolumn{2}{|l|}{ Rainfed } & $26.3 \mathrm{~b}$ & $38.3 \mathrm{~b}$ & $94.36 \mathrm{~b}$ & $5.67 \mathrm{a}$ \\
\hline \multicolumn{6}{|c|}{ HUMIC ACID (HA) } \\
\hline \multicolumn{2}{|l|}{0} & $26.0 \mathrm{~b}$ & $37.4 \mathrm{~b}$ & $94.54 \mathrm{c}$ & $5.50 \mathrm{a}$ \\
\hline \multicolumn{2}{|l|}{200} & $30.6 \mathrm{a}$ & $40.6 \mathrm{a}$ & $95.14 \mathrm{~b}$ & $4.85 \mathrm{~b}$ \\
\hline \multicolumn{2}{|l|}{400} & $30.3 \mathrm{a}$ & $40.6 \mathrm{a}$ & $95.71 \mathrm{a}$ & $4.34 \mathrm{~b}$ \\
\hline \multicolumn{6}{|c|}{ PGPR STRAIN (P) } \\
\hline \multicolumn{2}{|c|}{ Uninoculated } & $26.9 \mathrm{~b}$ & $38.0 \mathrm{~b}$ & $94.00 \mathrm{~b}$ & $6.05 \mathrm{a}$ \\
\hline \multicolumn{2}{|l|}{ OSU142 } & $30.2 \mathrm{a}$ & $40.4 \mathrm{a}$ & $95.70 \mathrm{a}$ & $4.36 \mathrm{~b}$ \\
\hline \multicolumn{2}{|l|}{ M3 } & $29.8 \mathrm{a}$ & $40.0 \mathrm{a}$ & $95.69 \mathrm{a}$ & $4.30 \mathrm{~b}$ \\
\hline \multicolumn{6}{|c|}{ SIGNIFICANCE } \\
\hline Source & df & \multicolumn{4}{|c|}{ Mean square } \\
\hline Year $(Y)$ & 1 & $0.68^{\mathrm{ns}}$ & $1.14^{\mathrm{ns}}$ & $1.18^{\mathrm{ns}}$ & $2.15^{\mathrm{ns}}$ \\
\hline $\mathrm{W}$ & 1 & $778.70^{* *}$ & $165.51^{* *}$ & $64.79^{* *}$ & $64.68^{* *}$ \\
\hline Y x W & 1 & $38.16^{*}$ & $0.63^{\mathrm{ns}}$ & $0.99^{\mathrm{ns}}$ & $0.98^{\mathrm{ns}}$ \\
\hline $\mathrm{HA}$ & 2 & $240.04^{* *}$ & $134.56^{* *}$ & $12.13^{* *}$ & $12.23^{* *}$ \\
\hline Y x HA & 2 & $6.31^{\mathrm{ns}}$ & $0.69^{\text {ns }}$ & $1.14^{\mathrm{ns}}$ & $1.46^{\mathrm{ns}}$ \\
\hline $\mathrm{P}$ & 2 & $113.34^{* *}$ & $58.55^{* *}$ & $34.49^{* *}$ & $35.71^{* *}$ \\
\hline$Y \times P$ & 2 & $11.35^{\mathrm{ns}}$ & $0.45^{\mathrm{ns}}$ & $4.22^{*}$ & $3.48^{\mathrm{ns}}$ \\
\hline W x HA & 2 & $35.76^{* *}$ & $9.67^{*}$ & $0.35^{\mathrm{ns}}$ & $0.19^{\mathrm{ns}}$ \\
\hline Y x W x HA & 2 & $9.04^{\mathrm{ns}}$ & $1.90^{\mathrm{ns}}$ & $0.43^{\text {ns }}$ & $1.71^{\mathrm{ns}}$ \\
\hline $\mathrm{W} \times \mathrm{P}$ & 2 & $20.82^{* *}$ & $0.06^{\mathrm{ns}}$ & $0.38^{\mathrm{ns}}$ & $0.13^{\mathrm{ns}}$ \\
\hline Y x W x P & 2 & $1.23^{\mathrm{ns}}$ & $3.77^{\mathrm{ns}}$ & $1.75^{\mathrm{ns}}$ & $0.91^{\mathrm{ns}}$ \\
\hline HA x P & 4 & $25.67^{* *}$ & $2.25^{\mathrm{ns}}$ & $0.91^{\mathrm{ns}}$ & $0.44^{\mathrm{ns}}$ \\
\hline Y x HA x P & 4 & $4.36^{\mathrm{ns}}$ & $1.34^{\mathrm{ns}}$ & $0.99^{\mathrm{ns}}$ & $0.67^{\mathrm{ns}}$ \\
\hline W $x$ HA $x$ P & 4 & $17.46^{* *}$ & $1.21^{\mathrm{ns}}$ & $1.55^{\mathrm{ns}}$ & $1.73^{\mathrm{ns}}$ \\
\hline \multicolumn{2}{|l|}{$\mathrm{CV}(\%)$} & 6.76 & 5.06 & 8.19 & 13.8 \\
\hline
\end{tabular}

The means were obtained from combined analysis of variance. ns, ${ }^{*}$, and ${ }^{* *}$ represent nonsignificant and significant at $\mathrm{P} \leq 0.05$ and $\mathrm{P} \leq 0.01$ according to DMRT, respectively. $\mathrm{CV}$ : coefficient of variations

Significant differences $(\mathrm{P}<0.01)$ in the 1000 seed weight, fertile and sterile seed percentage of safflower were observed for water regimes, HA, and bacterial inoculation (Table 5). A higher 1000 seed weight and fertile seed rate were noted for the irrigation treatment than for the rainfed condition. However, irrigation treatment induced a marked decline in sterile seed rate, with the lowest value noted for the rainfed 
conditions. On the other hand, all HA and PGPR treatments enhanced the 1000 seed weight by 8.6 and $6.3 \%$, respectively, over that of control (no HA or uninoculated). Similarly, fertile seed percentage significantly increased with increasing doses of HA and all the PGPR inoculations, whereas it considerably reduced the sterile seed percentage of safflower. The interactions between HA and PGPR were not significant for these parameters, but W x HA interaction was significant $(\mathrm{P}<0.05)$ for 1000 seed weight only (Table 5). The highest values for 1000 seed weight were noted for all the HA doses under irrigated condition, maintaining an increase from 8.7 to $11.0 \%$, compared to the control (Table 3).

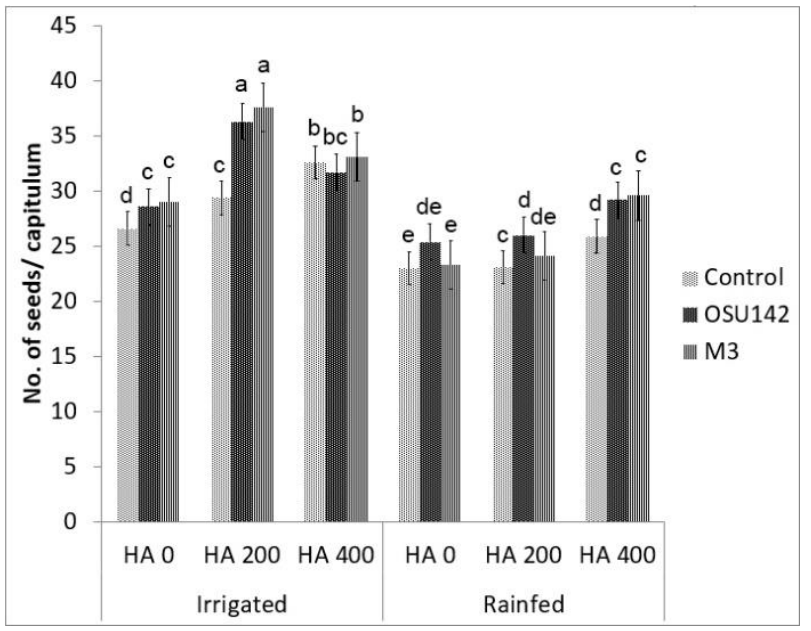

Figure 3. The interactive effect of water regimes, humic acids and PGPR on the number of seeds per capitulum of safflower. Different letters along with water regimes, humic acids and

PGPR treatments indicate significant differences in access treatment means from three replications tested at $P \leq 0.05$ according to DMRT

\section{Analysis of seed quality and yield traits}

Seed oil and protein contents were significantly affected by all the treatments (Table 6). Irrigation significantly $(\mathrm{P}<0.01$ and $\mathrm{P}<0.05)$ increased the seed oil and protein contents, respectively. Seed oil content significantly $(\mathrm{P}<0.01)$ increased with increasing HA doses, while the highest seed protein contents noted for both HA doses compared to control plants. Rhizobacteria inoculation significantly $(\mathrm{P}<0.01)$ increased seed oil and protein contents, with the highest oil content noted for M3 and with a higher protein content noted for OSU142 than for M3 and the uninoculated control.

Seed yield of safflower was significantly $(\mathrm{P}<0.01)$ affected by water regimes, HA, and rhizobacterial strains (Table 6). For different water regimes, irrigation treatment induced a marked increase in the seed yield, with the lowest value noted for the rainfed conditions. Likewise, increasing doses of HA and PGPR inoculation (OSU142 > M3) increased seed yield (Table 6). Moreover, W x HA, W x P, HA x P and W x HA x P $(\mathrm{P}<0.01)$ interactions were significant. The $\mathrm{W} \times \mathrm{HA}$ interaction (Table 3) revealed that the highest seed yield $\left(3.04 \mathrm{t} \mathrm{ha}^{-1}\right)$ was recorded for $400 \mathrm{~kg} \mathrm{HA} \mathrm{ha}^{-1}$ under irrigated condition, and the lowest yield $\left(1.02 \mathrm{t} \mathrm{ha}^{-1}\right)$ was recorded for the non-HA control under rainfed condition. The $400 \mathrm{~kg} \mathrm{HA} \mathrm{ha}^{-1}$ treatment significantly enhanced the seed yield by $60.8 \%$ and $83.3 \%$ under irrigated and rainfed condition, respectively, over that of 
their respective non-HA control. The $\mathrm{W} x \mathrm{P}$ interaction (Table 3) revealed that OSU142 was a potential strain for improving seed yield under both irrigated and rainfed conditions, with highest yield $\left(2.92 \mathrm{t} \mathrm{ha}^{-1}\right)$ noted for the irrigation treatment. Similarly, the HA x P interaction (Table 4) showed that OSU142 in all the HA rates considerably increased seed yield by 105.8 and $119.8 \%$, respectively, over that of the non-HA and uninoculated control. Moreover, the W x HA x P interaction (Fig. 4) revealed that the highest seed yield (3.37 $\left.\mathrm{t} \mathrm{ha}^{-1}\right)$ was recorded for $400 \mathrm{HA}+$ OSU142 treatment under irrigated conditions, with increase of $101.8 \%$ and followed by $400 \mathrm{HA}+\mathrm{M} 3(89.8 \%)$ treatment, compared to their respective control. However, the treatments of 400 HA + OSU14 $200 \mathrm{HA}+$ OSU142 and $400 \mathrm{HA}+\mathrm{M} 3$ were resulted for the higher seed yields (2.1 1.95 and $\left.1.91 \mathrm{t} \mathrm{ha}^{1}\right)$ under rainfed conditions, with increases of 186. 163.5 and $158.1 \%$, respectively, compared to their respective control.

Table 6. Seed yield and quality traits of safflower affected by humic acid application and bacterial inoculation under different water regimes

\begin{tabular}{|c|c|c|c|c|c|c|}
\hline & \multicolumn{2}{|c|}{ Seed content $(\%)$} & \multicolumn{3}{|c|}{ Yield (t ha-1) } \\
\hline & & Oil & Protein & Seed & Oil & Protein \\
\hline \multicolumn{7}{|c|}{ WATER REGIMES (W) } \\
\hline \multicolumn{2}{|l|}{ Irrigated } & $34.55 \mathrm{a}$ & $13.96 \mathrm{a}$ & $2.59 \mathrm{a}$ & $0.89 \mathrm{a}$ & $0.36 \mathrm{a}$ \\
\hline \multicolumn{2}{|l|}{ Rainfed } & $30.47 \mathrm{~b}$ & $13.47 \mathrm{~b}$ & $1.44 \mathrm{~b}$ & $0.44 \mathrm{~b}$ & $0.20 \mathrm{~b}$ \\
\hline \multicolumn{7}{|c|}{ HUMIC ACID (HA) } \\
\hline \multicolumn{2}{|l|}{0} & $30.64 \mathrm{c}$ & $12.75 \mathrm{~b}$ & $1.45 \mathrm{c}$ & $0.45 \mathrm{c}$ & $0.19 \mathrm{c}$ \\
\hline \multicolumn{2}{|l|}{200} & $32.93 \mathrm{~b}$ & $14.10 \mathrm{a}$ & $2.10 \mathrm{~b}$ & $0.70 \mathrm{~b}$ & $0.31 \mathrm{~b}$ \\
\hline \multicolumn{2}{|l|}{400} & $33.97 \mathrm{a}$ & $14.30 \mathrm{a}$ & $2.46 \mathrm{a}$ & $0.85 \mathrm{a}$ & $0.35 \mathrm{a}$ \\
\hline \multicolumn{7}{|c|}{ PGPR STRAIN (P) } \\
\hline \multicolumn{2}{|c|}{ Uninoculated } & $30.73 \mathrm{c}$ & $13.75 \mathrm{~b}$ & $1.62 \mathrm{c}$ & $0.51 \mathrm{c}$ & $0.23 \mathrm{c}$ \\
\hline \multicolumn{2}{|c|}{ OSU142 } & $32.93 \mathrm{~b}$ & $14.27 \mathrm{a}$ & $2.37 \mathrm{a}$ & $0.79 \mathrm{a}$ & $0.34 \mathrm{a}$ \\
\hline \multicolumn{2}{|l|}{ M3 } & $33.89 \mathrm{a}$ & $13.14 \mathrm{~b}$ & $2.08 \mathrm{~b}$ & $0.72 \mathrm{~b}$ & $0.28 \mathrm{~b}$ \\
\hline \multicolumn{7}{|c|}{ SIGNIFICANCE } \\
\hline Source & df & \multicolumn{5}{|c|}{ Mean square } \\
\hline Year (Y) & 1 & $21.78^{*}$ & $10.70^{\mathrm{ns}}$ & $77.48^{*}$ & $15.33^{*}$ & $3.51^{\mathrm{ns}}$ \\
\hline $\mathrm{W}$ & 1 & $450.18^{* *}$ & $6.35^{*}$ & $2665.11^{* *}$ & $436.21^{* *}$ & $54.57^{* *}$ \\
\hline Y x W & 1 & $0.83^{\mathrm{ns}}$ & $0.01^{\mathrm{ns}}$ & $0.57^{\mathrm{ns}}$ & $0.82^{\mathrm{ns}}$ & $0.14^{\mathrm{ns}}$ \\
\hline HA & 2 & $104.62^{* *}$ & $25.45^{* *}$ & $756.79^{* *}$ & $120.01^{* *}$ & $20.56^{* *}$ \\
\hline Y x HA & 2 & $0.08^{\mathrm{ns}}$ & $0.44^{\mathrm{ns}}$ & $0.59^{\mathrm{ns}}$ & $0.09^{\mathrm{ns}}$ & $0.006^{\mathrm{ns}}$ \\
\hline $\mathrm{P}$ & 2 & $94.44^{* *}$ & $11.75^{* *}$ & $372.89^{* *}$ & $58.81^{* *}$ & $7.78^{* *}$ \\
\hline $\mathrm{Y} \times \mathrm{P}$ & 2 & $0.24^{\mathrm{ns}}$ & $1.35^{\mathrm{ns}}$ & $0.15^{\mathrm{ns}}$ & $0.08^{\mathrm{ns}}$ & $0.10^{\mathrm{ns}}$ \\
\hline W x HA & 2 & $1.50^{\mathrm{ns}}$ & $0.94^{\mathrm{ns}}$ & $15.69^{* *}$ & $5.04^{* *}$ & $0.35^{\mathrm{ns}}$ \\
\hline $\mathrm{Y} \times \mathrm{W} \times \mathrm{HA}$ & 2 & $0.10^{\mathrm{ns}}$ & $0.72^{\text {ns }}$ & $0.21^{\mathrm{ns}}$ & $0.02^{\mathrm{ns}}$ & $0.03^{\mathrm{ns}}$ \\
\hline $\mathrm{W} \times \mathrm{P}$ & 2 & $3.49^{\mathrm{ns}}$ & $8.33^{*}$ & $14.53^{* *}$ & $2.50^{* *}$ & $0.36^{\mathrm{ns}}$ \\
\hline $\mathrm{Y} \times \mathrm{W} \times \mathrm{P}$ & 2 & $2.21^{\mathrm{ns}}$ & $1.35^{\mathrm{ns}}$ & $0.44^{\mathrm{ns}}$ & $0.26^{\mathrm{ns}}$ & $0.07^{\mathrm{ns}}$ \\
\hline $\mathrm{HA} \times \mathrm{P}$ & 4 & $1.14^{\mathrm{ns}}$ & $6.18^{*}$ & $10.21^{* *}$ & $1.83^{* *}$ & $0.06^{\mathrm{ns}}$ \\
\hline$Y \times H A x P$ & 4 & $0.72^{\mathrm{ns}}$ & $0.08^{\mathrm{ns}}$ & $0.75^{\mathrm{ns}}$ & $0.18^{\mathrm{ns}}$ & $0.02^{\mathrm{ns}}$ \\
\hline W $x$ HA $x$ P & 4 & $0.17^{\mathrm{ns}}$ & $0.39^{\text {ns }}$ & $5.84^{* *}$ & $1.42^{* *}$ & $0.17^{\mathrm{ns}}$ \\
\hline $\mathrm{CV}(\%)$ & & 5.95 & 8.86 & 5.53 & 7.54 & 9.15 \\
\hline
\end{tabular}

The means were obtained from combined analysis of variance. ns, *, and ${ }^{* *}$ represent nonsignificant and significant at $\mathrm{P} \leq 0.05$ and $\mathrm{P} \leq 0.01$ according to DMRT, respectively. $\mathrm{CV}$ : coefficient of variations 


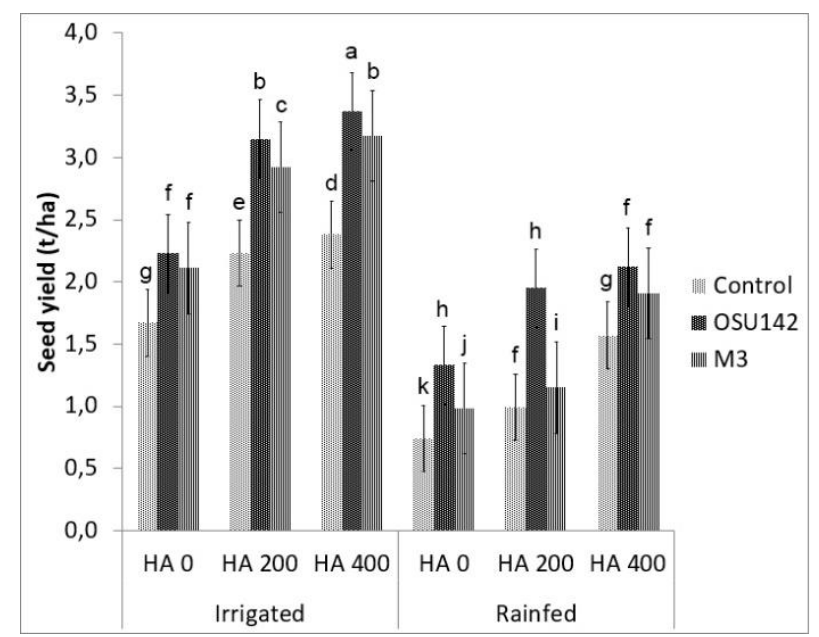

Figure 4. The interactive effect of water regimes, humic acids and PGPR on the seed yield $\left(t h a^{-1}\right)$ of safflower. Different letters along with water regimes, humic acids and PGPR treatments indicate significant differences in access treatment means from three replications tested at $P \leq 0.05$ according to DMRT

Results for the oil and protein yields showed trends similar to that seen for seed yield. Significant differences $(\mathrm{P}<0.01)$ in the oil and protein yields of safflower were observed for water regimes, HA, and bacterial inoculation (Table 6). For different water regimes, the highest oil and protein yields were recorded for irrigated condition with 0.89 and $0.36 \mathrm{t}^{-}$respectively. The increasing doses of HA (400 HA > $\left.200 \mathrm{HA}\right)$ and PGPR inoculation (OSU142 > M3) significantly $(\mathrm{P}<0.01)$ improved oil and protein yields (Table 6). Furthermore, significant $(\mathrm{P}<0.01)$ interactions were observed in $\mathrm{W} \mathrm{x}$ $\mathrm{HA}, \mathrm{W} \times \mathrm{P}, \mathrm{HA} \times \mathrm{P}$ and $\mathrm{W} \times \mathrm{HA} \times \mathrm{P}$ for oil yield. The $\mathrm{W} \times \mathrm{HA}$ interaction (Table 3) showed that the highest oil yield $\left(1.10 \mathrm{t} \mathrm{ha}^{-1}\right)$ was recorded for $400 \mathrm{~kg} \mathrm{HA} \mathrm{ha}^{-1}$ under irrigated condition, and the lowest yield $\left(0.29 \mathrm{t} \mathrm{ha}^{-1}\right)$ was recorded for the non-HA control under rainfed condition. Likewise, the $\mathrm{W} x \mathrm{P}$ interaction (Table 3) revealed that OSU142 and M3 strains enhanced oil yield under both irrigated and rainfed conditions, with highest yield (1.01 and $0.98 \mathrm{t} \mathrm{ha}^{-}$respectively) noted for the irrigation treatment. Furthermore, the HA x P interaction (Table 4) showed that the highest oil yield was observed for $400 \mathrm{HA}+$ OSU142 treatment. Moreover, the W x HA x P interaction (Fig. 5) revealed that the highest oil yields were recorded for $400 \mathrm{HA}+$ OSU14 $400 \mathrm{HA}$ + M3 and $200 \mathrm{HA}+$ OSU142 treatments under irrigated conditions, with increases of 127. 119.1 and $108.4 \%$, compared to their respective control, whereas the treatments of $400 \mathrm{HA}+\mathrm{M} 3$ and $400 \mathrm{HA}+\mathrm{OSU} 142$ were resulted in the higher seed yields under rainfed conditions, with increases of 257.1 and $226.5 \%$, respectively, compared to their respective control.

\section{Elemental analysis of safflower seeds}

Macro- and micronutrients contents (P, K, Ca, Mg, Fe, Zn, Cu and Mn) of safflower seeds were significantly $(\mathrm{P}<0.01)$ affected by water regimes, HA, and PGPR strains (Table 7). A higher $\mathrm{P}, \mathrm{K}, \mathrm{Ca}, \mathrm{Fe}, \mathrm{Cu}$ and $\mathrm{Mn}$ contents were noted for the irrigation treatment than for the rainfed condition, whereas rainfed condition induced a marked increase in $\mathrm{Mg}$ and $\mathrm{Zn}$ contents, with the lowest value noted for the irrigation treatment. 
However, the seeds in both HA and PGPR treated plants generally had higher macroand micronutrient contents compared to the control (no HA or uninoculated) plants.

Table 7. Mineral composition ( $\mathrm{mg} \mathrm{kg}^{-1}$ ) of safflower seeds affected by humic acid application and bacterial inoculation under different water regimes

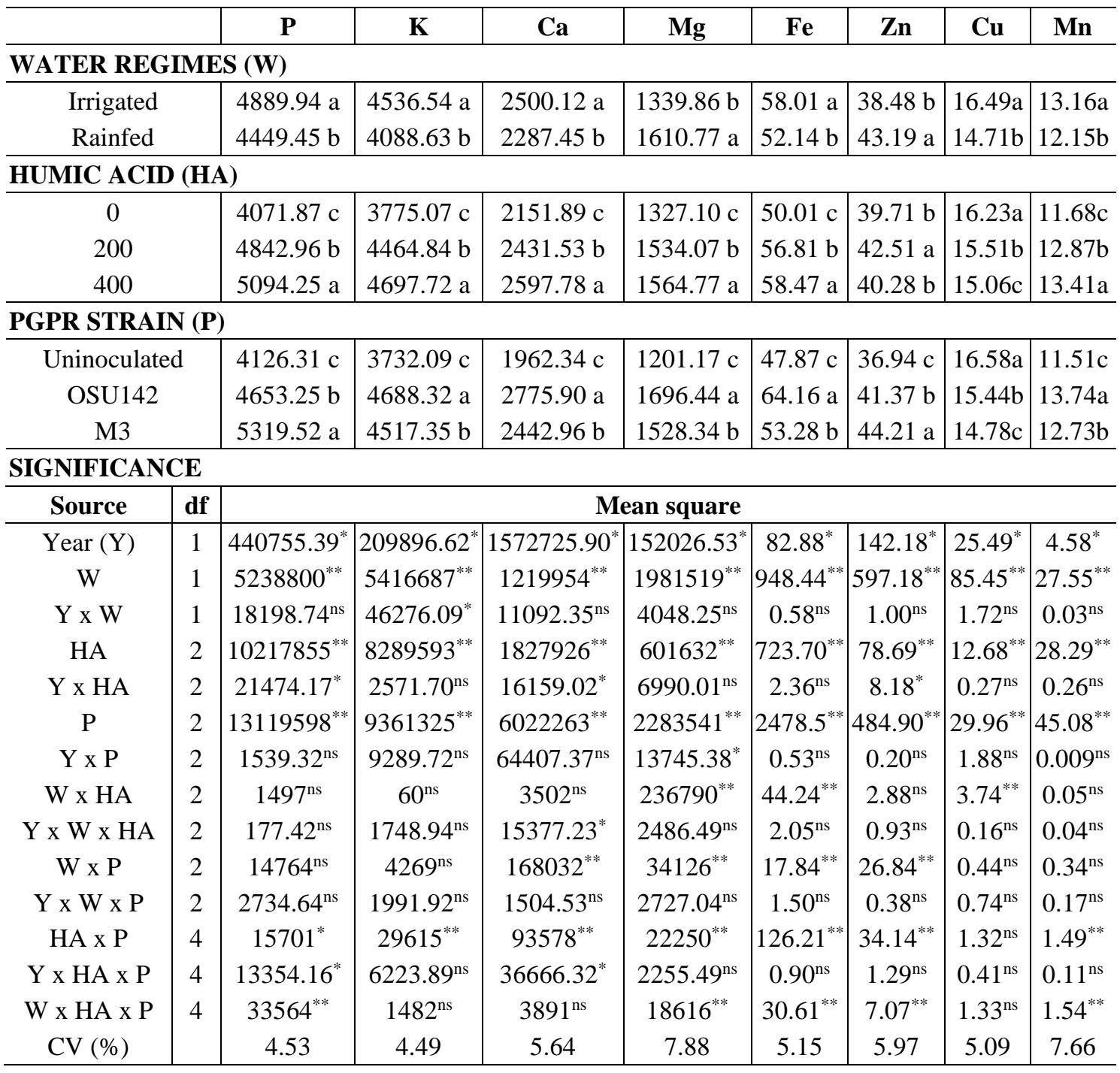

The means were obtained from combined analysis of variance. ns, ${ }^{*}$, and ${ }^{* *}$ represent nonsignificant and significant at $\mathrm{P} \leq 0.05$ and $\mathrm{P} \leq 0.01$ according to DMRT, respectively. $\mathrm{CV}$ : coefficient of variations

For macronutrient contents $(\mathrm{P}, \mathrm{K}, \mathrm{Ca}$ and $\mathrm{Mg}$ ), the individual effects of all treatments were significant $(\mathrm{P}<0.0$ Table 7$)$. All macronutrient contents increased with increasing doses of HA (400 HA > $200 \mathrm{HA}$ ), whereas the M3 inoculation noted for the highest P content, and the OSU142 inoculation noted for the highest $\mathrm{K}, \mathrm{Ca}$ and $\mathrm{Mg}$ contents. For these parameters, significant interactions were observed in $\mathrm{W} \times \mathrm{HA}(\mathrm{P}<0.01)$ for $\mathrm{Mg}$ content, $\mathrm{W} \times \mathrm{P}(\mathrm{P}<0.01)$ for $\mathrm{Ca}$ and $\mathrm{Mg}$ contents, $\mathrm{HA} \times \mathrm{P}(\mathrm{P}<0.01$ and $\mathrm{P}<0.05)$ for all the macronutrients, and $\mathrm{W} \times \mathrm{HA} \times \mathrm{P}(\mathrm{P}<0.01)$ for $\mathrm{P}$ and $\mathrm{Mg}$ contents (Table 7). The $\mathrm{W} \times$ HA interaction (Table 8) showed that the highest $\mathrm{Mg}$ content $\left(1750.60 \mathrm{mg} \mathrm{kg}^{-1}\right)$ was recorded for $200 \mathrm{~kg} \mathrm{HA} \mathrm{ha}{ }^{-1}$ under rainfed condition, and the lowest value (1272.78 $\mathrm{mg} \mathrm{kg}^{-1}$ ) was recorded for the non-HA control under irrigated condition. 


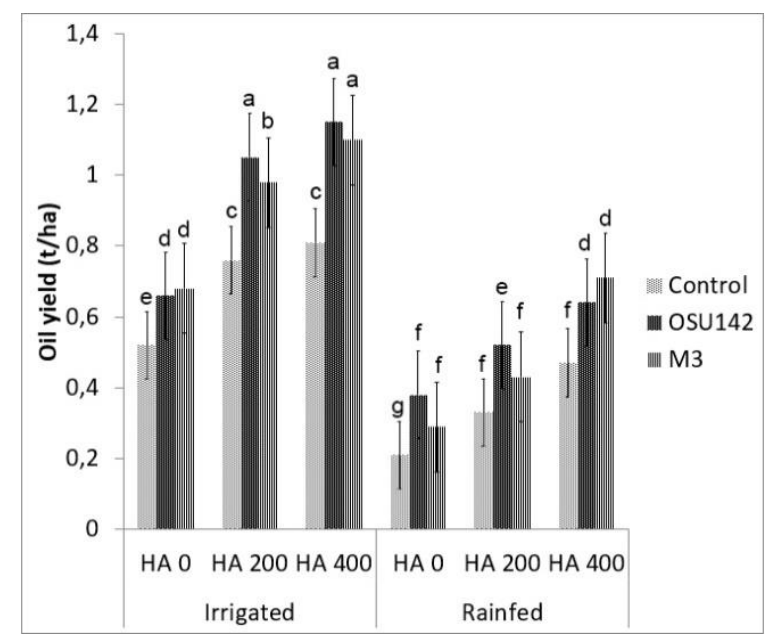

Figure 5. The interactive effect of water regimes, humic acids and PGPR on the oil yield ( $\left.t \mathrm{ha}^{-1}\right)$ of safflower. Different letters along with water regimes, humic acids and PGPR treatments indicate significant differences in access treatment means from three replications tested at $P \leq 0.05$ according to DMRT

Table 8. Interaction between humic acid (HA) water regimes (Irrigated and Rainfed); PGPR strain and water regimes on nutrient content $\left(\mathrm{mg} \mathrm{kg}^{-1}\right)$ of safflower seeds

\begin{tabular}{|c|c|c|c|c|c|c|c|c|c|c|}
\hline \multirow{2}{*}{ Factors } & \multicolumn{2}{|c|}{ Calcium } & \multicolumn{2}{|c|}{ Magnesium } & \multicolumn{2}{|c|}{ Iron } & \multicolumn{2}{|c|}{ Zinc } & \multicolumn{2}{|c|}{ Copper } \\
\hline & $\mathbf{I}$ & $\mathbf{R}$ & I & $\mathbf{R}$ & I & $\mathbf{R}$ & $\mathbf{I}$ & $\mathbf{R}$ & I & $\mathbf{R}$ \\
\hline \multicolumn{11}{|l|}{ HA $\left(\mathrm{kg} \mathrm{ha}^{-1}\right)$} \\
\hline 0 & 2074.44 & 2817.22 & $1272.78^{\mathrm{f}}$ & $1381.41^{\mathrm{d}}$ & $54.25^{\mathrm{d}}$ & $45.78^{\mathrm{e}}$ & 37.14 & 42.29 & $17.06^{\mathrm{a}}$ & $15.40^{\mathrm{c}}$ \\
\hline 200 & 2520.21 & 3296.56 & $1317.56^{\mathrm{e}}$ & $1750.60^{\mathrm{a}}$ & $59.27^{b}$ & $54.36^{\mathrm{d}}$ & 40.07 & 44.95 & $16.11^{b}$ & $14.92^{\mathrm{c}}$ \\
\hline 400 & 2738.88 & 3481.12 & $1429.25^{\mathrm{c}}$ & $1700.29^{b}$ & $60.68^{a}$ & $56.28^{c}$ & 38.25 & 42.32 & $16.30^{\mathrm{b}}$ & $13.82^{\mathrm{d}}$ \\
\hline \multicolumn{11}{|c|}{ PGPR STRAIN } \\
\hline Uninoculated & $2133.98^{\mathrm{e}}$ & $1790.70^{\mathrm{f}}$ & $1100.67^{\mathrm{f}}$ & $1301.66^{\mathrm{e}}$ & $51.65^{\mathrm{d}}$ & $44.10^{\mathrm{f}}$ & $33.71^{\mathrm{e}}$ & $40.16^{\mathrm{d}}$ & 17.54 & 15.63 \\
\hline OSU142 & $2811.25^{\mathrm{a}}$ & $2740.54^{b}$ & $1549.11^{\mathrm{c}}$ & $1843.75^{\mathrm{a}}$ & $66.74^{\mathrm{a}}$ & $61.59^{b}$ & $39.87^{d}$ & $42.87^{b}$ & 16.20 & 14.68 \\
\hline M3 & $2554.81^{c}$ & $2331.11 \mathrm{~d}$ & $1369.80^{\mathrm{d}}$ & $1686.88^{b}$ & $55.82^{\circ}$ & $50.74 \mathrm{e}$ & $41.89^{\mathrm{c}}$ & $46.55^{a}$ & 15.74 & 13.83 \\
\hline
\end{tabular}

Means having different letters are significantly different at $\mathrm{P} \leq 0.05$ according to DMRT

Likewise, the W x P interaction (Table 8) revealed that OSU142 strains enhanced Ca content of seeds under both irrigated and rainfed conditions, with highest value $\left(2811.25 \mathrm{mg} \mathrm{kg}^{-1}\right)$ noted for the irrigation treatment, whereas the highest $\mathrm{Mg}$ content of seeds (1843.75 mg kg${ }^{-1}$ ) noted for OSU142 inoculation under rainfed conditions. Furthermore, the HA x P interaction (Table 9) showed that the highest $\mathrm{K}, \mathrm{Ca}$ and $\mathrm{Mg}$ contents were observed for $400 \mathrm{HA}+$ OSU142 treatment, whereas the highest P content noted for $400 \mathrm{HA}+\mathrm{M} 3$ treatment.

Moreover, the W x HA x P interactions revealed that the highest phosphorus content was recorded for $400 \mathrm{HA}+\mathrm{M} 3$ under irrigated conditions, with increase of $56.3 \%$, whereas the highest $\mathrm{Mg}$ content was recorded for $400 \mathrm{HA}+$ OSU142 under rainfed conditions, with increase of $96.6 \%$, compared to their respective control (Fig. $6 a$ and $b$ ). 
Table 9. Interaction between humic acid (HA) and PGPR strain on nutrient content ( $\mathrm{mg} \mathrm{kg}^{-1}$ ) of safflower seeds

\begin{tabular}{c|c|c|c|c|c|c|c|c}
\hline $\begin{array}{c}\text { HA } \\
\left.\text { (kg ha-1 }^{-1}\right)\end{array}$ & PGPR strain & $\mathbf{P}$ & $\mathbf{K}$ & $\mathbf{M g}$ & $\mathbf{C a}$ & $\mathbf{F e}$ & $\mathbf{Z n}$ & $\mathbf{M n}$ \\
\hline \multirow{3}{*}{0} & Uninoculated & $3523.43 \mathrm{~h}$ & $3207.32 \mathrm{~h}$ & $1001.32 \mathrm{f}$ & $1627.45 \mathrm{~g}$ & $38.48 \mathrm{~h}$ & $33.97 \mathrm{f}$ & $10.09 \mathrm{~d}$ \\
& OSU142 & $3938.49 \mathrm{~g}$ & $4108.40 \mathrm{e}$ & $1575.24 \mathrm{c}$ & $2629.59 \mathrm{c}$ & $61.26 \mathrm{c}$ & $41.12 \mathrm{c}$ & $13.02 \mathrm{~b}$ \\
& M3 & $4753.68 \mathrm{~d}$ & $4009.47 \mathrm{f}$ & $1402.71 \mathrm{~d}$ & $2198.62 \mathrm{e}$ & $50.31 \mathrm{~g}$ & $44.08 \mathrm{~b}$ & $11.93 \mathrm{c}$ \\
\hline \multirow{3}{*}{200} & Uninoculated & $4274.58 \mathrm{f}$ & $3912.74 \mathrm{~g}$ & $1303.02 \mathrm{e}$ & $2082.94 \mathrm{f}$ & $51.46 \mathrm{f}$ & $39.03 \mathrm{~d}$ & $12.07 \mathrm{c}$ \\
& OSU142 & $4755.06 \mathrm{~d}$ & $4866.69 \mathrm{c}$ & $1720.93 \mathrm{~b}$ & $2735.98 \mathrm{~b}$ & $65.10 \mathrm{~b}$ & $42.00 \mathrm{c}$ & $13.70 \mathrm{~b}$ \\
& M3 & $5499.22 \mathrm{~b}$ & $4615.10 \mathrm{~d}$ & $1578.16 \mathrm{c}$ & $2475.66 \mathrm{~d}$ & $53.90 \mathrm{e}$ & $46.51 \mathrm{a}$ & $12.88 \mathrm{~b}$ \\
\hline \multirow{3}{*}{400} & Uninoculated & $4580.91 \mathrm{e}$ & $4076.21 \mathrm{e}$ & $1299.14 \mathrm{e}$ & $2176.64 \mathrm{e}$ & $53.68 \mathrm{e}$ & $37.82 \mathrm{e}$ & $12.35 \mathrm{c}$ \\
& OSU142 & $4996.18 \mathrm{c}$ & $5089.85 \mathrm{a}$ & $1791.13 \mathrm{a}$ & $2962.11 \mathrm{a}$ & $66.14 \mathrm{a}$ & $40.97 \mathrm{c}$ & $14.50 \mathrm{a}$ \\
& M3 & $5705.65 \mathrm{a}$ & $4927.46 \mathrm{~b}$ & $1604.04 \mathrm{c}$ & $2654.58 \mathrm{c}$ & $55.61 \mathrm{~d}$ & $47.07 \mathrm{c}$ & $13.37 \mathrm{~b}$ \\
\hline
\end{tabular}

Means having different letters are significantly different at $\mathrm{P} \leq 0.05$ according to DMRT

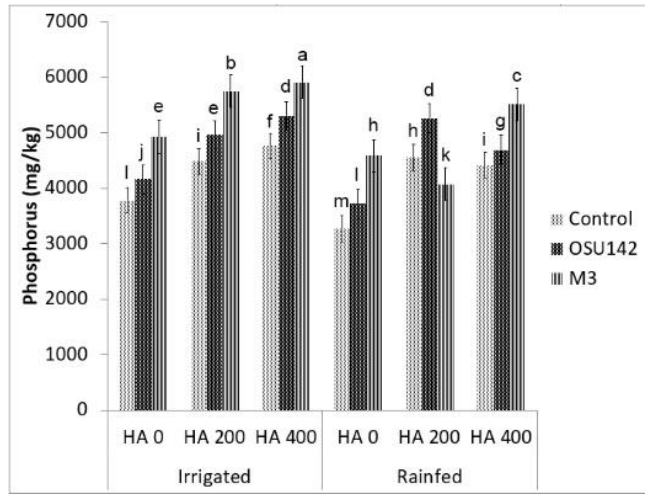

(a)

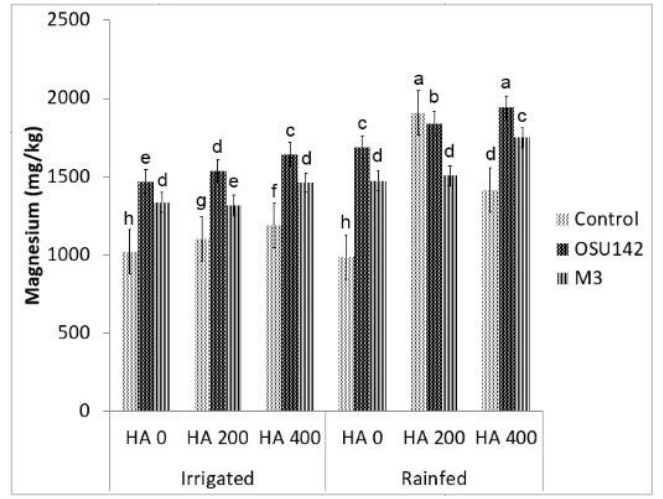

(b)

Figure 6. Phosphorus content $\left(\mathrm{mg} \mathrm{kg}^{-1}\right)\left(\right.$ a) and magnesium content $\left(\mathrm{mg} \mathrm{kg}^{-1}\right)(b)$ in seed at humic acid treatments and rhizobacterial inoculation and their interaction under the irrigated and rainfed conditions. Different letters along with water regimes, humic acids and PGPR treatments indicate significant differences in access treatment means from three replications tested at $P \leq 0.05$ according to DMRT

For micronutrient contents (iron, zinc, copper and manganese), the individual effects of all treatments were significant $(\mathrm{P}<0.0$ Table 7$)$. Iron and manganese contents increased with increasing doses of HA (400 HA > $200 \mathrm{HA})$, whereas the highest zinc and copper contents noted for $200 \mathrm{~kg} \mathrm{HA} \mathrm{ha}^{-1}$ and non-HA control, respectively. The OSU142 and M3 inoculations significantly $(\mathrm{P}<0.01)$ increased contents of iron, zinc and manganese, whereas it marked decline in copper content with the highest value noted for the uninoculated control. For these parameters, significant $(\mathrm{P}<0.01)$ interactions were observed in $\mathrm{W} \times \mathrm{HA}$ for iron and copper contents, $\mathrm{W} \times \mathrm{P}$ for iron and zinc contents, HA x P for iron, zinc and manganese contents, W x HA x P for iron, zinc and manganese contents (Table 7). The W x HA interaction (Table 8) showed that the highest iron $\left(60.68 \mathrm{mg} \mathrm{kg}^{-1}\right)$ and the lowest copper $\left(13.82 \mathrm{mg} \mathrm{kg}^{-1}\right)$ contents were recorded for $400 \mathrm{~kg} \mathrm{HA} \mathrm{ha}^{-1}$ under irrigated condition, whereas the highest copper content (17.06 mg kg-1) was recorded for the non-HA control under irrigated condition. 
Likewise, the W x P interaction (Table 8) revealed that OSU142 strains improved iron content of seeds under both irrigated and rainfed conditions, with highest value (66.74 $\left.\mathrm{mg} \mathrm{kg}^{-1}\right)$ noted for the irrigation treatment, whereas the highest zinc content of seeds $\left(46.55 \mathrm{mg} \mathrm{kg}^{-1}\right)$ noted for M3 inoculation under rainfed conditions. Furthermore, the HA x P interaction (Table 9) indicated that the highest iron and manganese contents were observed for $400 \mathrm{HA}+$ OSU142 treatment, whereas the highest zinc content noted for $200 \mathrm{HA}+\mathrm{M} 3$ treatment. Similarly, the W x HA x P interactions (Fig. 7a, $b$ and $c$ ) revealed that the highest zinc content was recorded for $200 \mathrm{HA}+$ OSU142 under rainfed condition, whereas the highest iron and manganese contents were recorded for 400 HA + OSU142 under irrigated conditions, with increases of 51.3 and $41.9 \%$, respectively, compared to their respective controls.

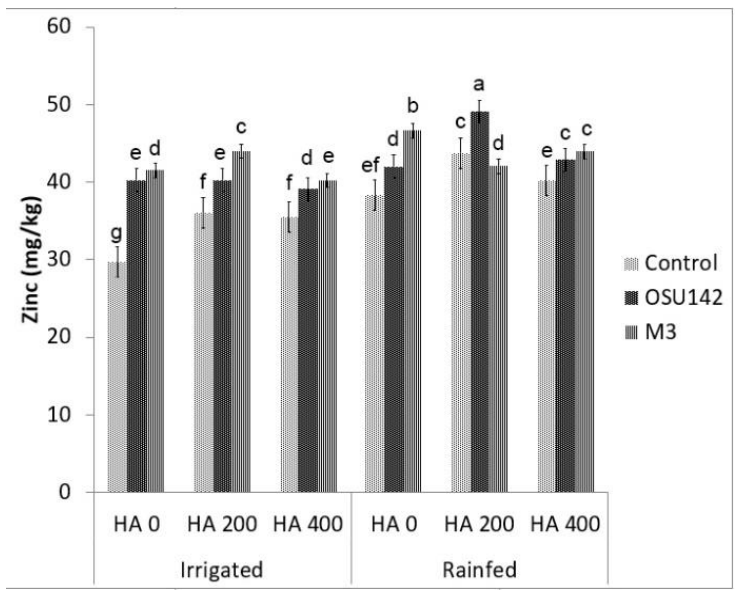

(a)

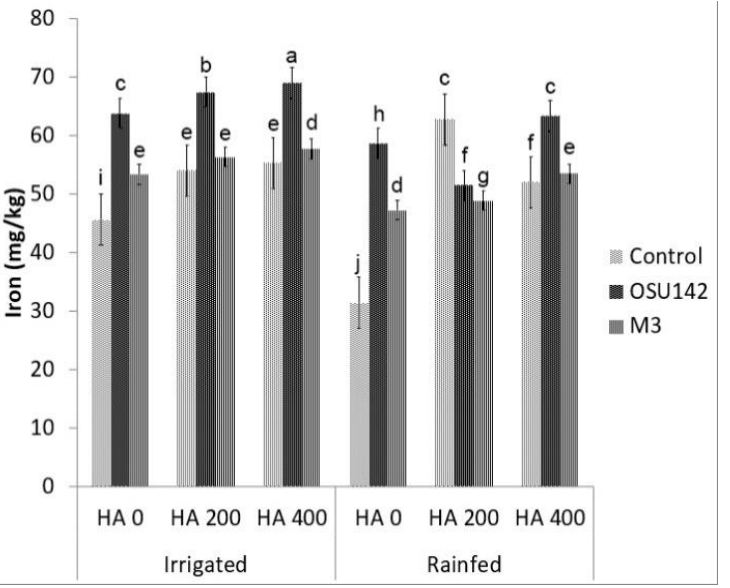

(b)

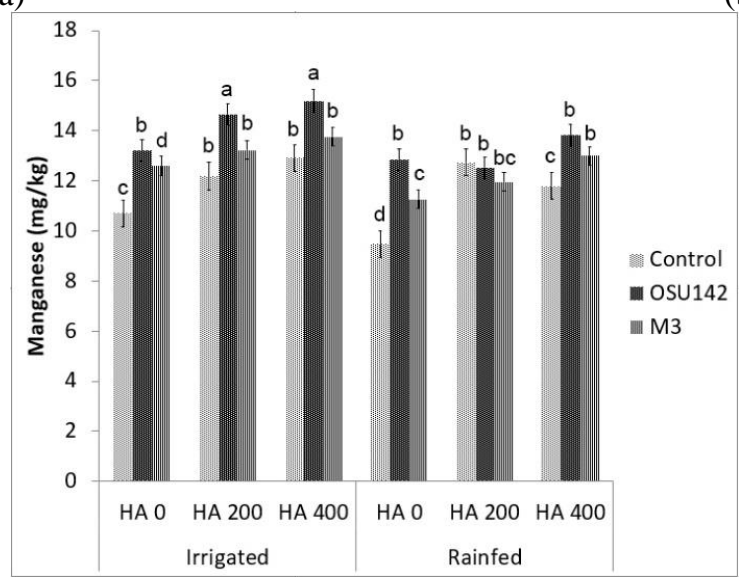

(c)

Figure 7. Zinc content $\left(m g \mathrm{~kg}^{-1}\right)(a)$, iron content $\left(m g \mathrm{~kg}^{-1}\right)(\mathrm{b})$, manganese content $\left(m g \mathrm{~kg}^{-1}\right)(\mathrm{c})$ in seed at humic acid treatments and rhizobacterial inoculation and their interaction under the irrigated and rainfed conditions. Different letters along with water regimes, humic acids and $P G P R$ treatments indicate significant differences in access treatment means from three replications tested at $P \leq 0.05$ according to DMRT

\section{Discussion}

In this two-year field study, humic acid (200 and $400 \mathrm{~kg} \mathrm{ha}^{-1}$ ) and two rhizobacterial strains (Bacillus megatorium M3 and Bacillus subtilis OSU-142) were evaluated for 
their effect on the growth, yield and quality of safflower under irrigated and rainfed conditions. The results indicated that humic acid and rhizobacterial inoculation under irrigated conditions significantly increased the growth and yield of safflower. However, the combined application of rhizobacterial strains and humic acid alleviated the negative effects of water deficit on growth and yield of safflower under rainfed conditions, thereby presented an improved strategy for the remediation of water stress in the safflower production in arid and semi-arid regions.

Nowadays, worldwide studies are intensively maintained to isolate and characterize the PGPR strains for improving crop production in sustainable agriculture and several stress conditions. Many PGPR species, including Azotobacter, Azosprillum, Bacillus, Serratia, Enterobacter, Pseudomonas, Klebsiella and Variovorax etc., improve plant growth by releasing of plant growth stimulating compounds (e.g. phytohormones such as IAA, cytokines or auxins) or improving in nutrient uptakes (e.g. $\mathrm{N}_{2}$ fixing, Psolubilizing or siderophore production) or enhancing in plant tolerance to both biotic and abiotic stresses (Glick, 1995; Nelson, 2004; Kristin and Miranda, 2013; Backer et al., 2018). In the present study, OSU-142 (Bacillus subtilis) and M3 (Bacillus megatorium) strains, having properties of which were mentioned above, were tested for agronomic performance of safflower in the field conditions. Between the tested two bacteria, the $\mathrm{N}_{2}$-fixing OSU-142 strain was determined the most effective in growth promotion (esp. plant height, stem and capitilum diameters, and number of capitula), and yields of seed, oil and protein in safflower. It was observed no difference between bacteria in terms of seed characteristics (e.g. number of seeds, 1000 seed weight, and fertile and sterile seed ratios) of safflower, whereas P-solubilizing M3 strain was showed the greatest performance in oil content improvement of seeds. Both of these strains have IAA and ACC deaminase production (Cakmakci et al., 200 2006), and these properties might be the cause of the noted plant growth promotion (Arshad et al., 2008; Glick, 2014; Vejan et al., 2016; Chandra et al., 2019). This potential relationship is supported by Belimov et al. (2009) and Chandra et al. (2019) who observed that inoculations of pea and wheat with Variovorax sp., possessing IAA, ACC deaminase, and $\mathrm{N}_{2}$-fixing and P-solubilizing activities, significantly increased the shoot biomass of pea and yield characteristics of wheat. Similar effects of PGPR inoculation on plant growth promotion have been observed in the several studies in sugar beet and barley (Cakmakci et al., 200 2006), sugarcane (Silva et al., 2017), sunflower (Shadid et al., 2012), wheat (Rosas et al., 2009; Hungria et al., 2010; Rana et al., 2012; Chandra et al., 2019), rice (Lucas et al., 2009), bean (Hoyos-Carvajal et al., 2009), canola (El-Howeity and Asfour, 2012), maize (Thonar et al., 2017) and soy (Cassán et al., 2009). Moreover, the superior performance of OSU14 compared to that of $\mathrm{M}$ could be due to its $\mathrm{N}_{2}$-fixing ability converting atmospheric nitrogen $\left(\mathrm{N}_{2}\right)$ into ammonia $\left(\mathrm{NH}_{3}\right)$ or related nitrogenous compounds in soil, which helps the plant in nitrogen acquisition (Cakmakci et al., 200 2006). Additionally, biological nitrogen fixation is considered to be an essential to plants because fixed inorganic nitrogen compounds are required for the biosynthesis of all nitrogen-containing organic compounds, such as amino acids and proteins, nucleoside triphosphates and nucleic acids. Hence, the greater nitrogen uptake by plants directly affects plant growth. In the study, the effect of M3 strain showing the higher performance in increasing oil contents of safflower seed could be due to its phosphate solubilizing ability, which one of the most important traits associated with plant phosphate nutrition. The previous studies also indicated that enhanced phosphate acquisition by phosphate solubilizing microorganisms positively affect seed oil 
concentration of oilseed crops (Mirzakhani et al., 2009; Shahid et al., 2012; Sharifi et al., 2017; Nosheen et al., 2018; Chandra et al., 2019).

In the current study, the humic acid and bacterial inoculation considerably increased the morphologic, seed and yield characteristics of safflower in the field conditions. The results revealed that morphologic characteristics, such as plant height, stem diameter, numbers of branches and capitula, and capitulum diameter, and yields of seed, oil and protein were significantly affected by both humic acid and bacterial inoculation, thus their highest values obtained from $400 \mathrm{~kg} \mathrm{HA} \mathrm{ha}^{-1}$ and OSU142 strain, respectively. Similarly, the seed characteristics and seed protein concentration of safflower treated by humic acid and bacterial inoculation significantly increased compared to control, but showed no difference between HA doses or PGPR strains. Overall, the enhanced safflower growth due to humic acids and PGPR might be attributed to their beneficial effects on the plant growth, which affects because of promotion of root morphology, nutrient uptake, and soil biological activity etc. (Schmidt et al., 2007; Puglisi et al., 2013; Canellas and Olivares, 2014). The plants treated with $400 \mathrm{~kg} \mathrm{HA} \mathrm{ha}^{-1}$ exhibited considerably increased morphologic characteristics of safflower, which probably directly affected the root growth, especially lateral root emergence (Nardi et al., 2009), and indirectly improved photosynthetic rate, leading to increased growth and yield of safflower (Puglisi et al., 2013; Canellas and Olivares, 2014). This proposed explanation is supported by those of Canellas et al. (2013), who noted significantly enhanced the rate of net photosynthesis in maize in response to the increase of the humate concentration under greenhouse condition. Similarly, different endophytic rhizobacteria species have been also reported that increased the photosynthetic rate, stomatal conductance, transpiration velocity, water utilization efficiency in rice, which might support plant growth improvement (Chi et al., 2005). More recently, Schoebitz et al. (2016), Esringu et al. (2016) and Olivares et al. (2015) have reported significant growth and yield improvements in blueberry, Hungarian vetch and tomato, respectively, due to humates and PGPR inoculation under field conditions.

Although the studies recently reviewed basic mechanisms and synergistic effects produced by integrated use of humic substances and PGPR on nutrient uptake, growth and yield of various crops under different stress conditions (Baldatto et al., 2010; Glick, 2014; Esringu et al., 2016; Schoebitz et al., 2016; Silva et al., 2017; Olivares et al., 2017), their effects has not been previously reported in safflower under different water regimes. In the study, the enhanced growth and yield of safflower might be due to similar synergistic effects of humic acids and PGPR under irrigated and rainfed conditions. Similar to the present results, humic substances widely reported to having potential as enhancer of PGPR efficiency (Esringu et al., 2016; Schoebitz et al., 2016; Silva et al., 2017). Cakmakci et al. (2006) observed a positive correlation among soil organic matter content, PGPR strains and growing stage, and reported that the effect of PGPR was greater in the high soil organic matter content, and at early plant growth stages than at the later. In the previous studies, it has also been notified that the combined use of PGPR and humic substances increased productions of maize grains by $65 \%$, tomato fruits by $87.1 \%$ and potato tubers by $140 \%$ under field conditions (Canellas et al., 2013; Olivares et al., 2015; Ekin, 2019).

Safflower is an extensively branching oilseed plant, and dry matter accumulation in connection with seed yield depends not only on plant height but also on numbers of branch and capitula, and some morphological characteristics sensitive to water deficit stress (Dajue and Mündel, 1996). In the current study, the plant height, number of 
capitula per plant and number of seeds per capitulum were the highest for safflower plants treated with integrated use of humic acids and PGPR under irrigated conditions. However, their effects noted for relatively higher for safflower yield characteristics than for irrigated plants under rainfed conditions. Under rainfed conditions, the combined use of humic acid and PGPR helped the safflower plants acclimatize to water-deficit (rainfed) conditions by improving a variety of morphologic and yield characteristics, including plant height, stem diameter, number of capitula, capitilum diameter, number of seeds, 1000 seed weight and fertile seed percentage, which cause for enhancing seed and oil yields. The improved safflower growth and yield by bacterial inoculation and humic acids under rainfed conditions might be correlated with the enhancement in plant tolerance to drought by increasing their water content, which can be attributed to the enhancement of root growth by humic substances and PGPR inoculation. In addition, it has also been reported that PGPR could enhance the plant growth under stress conditions by biosynthesis of phytohormones (IAA, GA, and cytokines), producing the enzyme ACC deaminase, fixing asymbiotic nitrogen, and solubilizing of phosphates and other nutrient elements (Glick, 2014). This proposed explanation is consistent with the results of Sahin et al. (2015), who stated $\mathrm{N}_{2}$-fixing, P-solubilization, and producing of IAA, GA and SA of PGPR has been basic factors improving nutrient uptake and growth of lettuce. They also reported that PGPR inoculation significantly increased growth and yield of lettuce plants under lower and well-watered conditions, and alleviated the harmful effects of lower irrigation conditions. Similar results were noted by Rubin et al. (2017) and Mutumba et al. (2018), who reported that plants were highly responsive to bacterial inoculation under well-watered conditions, but the effect was relatively higher under drought conditions. Other previous studies have also reported improving effects of PGPR inoculation on the yield and growth of different crops grown under drought stress (Vivas et al., 2003; Arkhipova et al., 2007; Marulanda et al., 2009; Sandhya et al., 2010).

In the current study, the interactive effect of humic acids and PGPR inoculation on nutrient contents of safflower seeds was studied in the natural field conditions. In general, the nutrient uptake of safflower plants except for $\mathrm{Mg}$ and $\mathrm{Zn}$ contents was lower in rainfed conditions, compared to the irrigated conditions. However, humic acids and PGPR inoculation considerable improved macro and micro nutrient contents of seeds except copper under both irrigated and rainfed conditions. In addition, $\mathrm{K}, \mathrm{Ca}, \mathrm{Mg}$, $\mathrm{Fe}$ and Mn concentrations of the seeds noted for the highest for plants treated with OSU142 strain, whereas P and Zn concentrations in the M3 strain. In the study, the specialty of M3 strain highly responded to enhancing especially phosphorus and zinc uptake could be attributed to its P-solubilizing ability and affecting root growth and lateral root formation by producing plant hormones such as IAA in the rhizosphere (Cakmakci et al., 2001; Chandra et al., 2019). Likewise, $\mathrm{N}_{2^{-}}$fixing ability and phytohormone production of OSU142 strain also might have been one of the main factors improving growth and mineral nutrient uptake of safflower. Moreover, the combined use of humic acids and PGPR also alleviated the harmful effects of water deficit conditions on the mineral nutrient uptake. Under rainfed conditions, the reduction in nutrient uptake may be due to a notable decrease in root cation exchange capacity of the plants, which can accumulate some ions, organic and amino acids to avoid harmful effects of abiotic stress conditions in dry environments (Sahin, 2015). Similarly, the reduction in copper content may be attributed to strongly retain in the soil after the application of humic acids (Rong et al., 2020). Nowadays, the applications of 
humic acid and PGPR have been widely used to improve soil functions, to mobilize or immobilize cations and metal ions in soil, leading to increased nutrient uptake from soils (Rubin et al., 2017; Rong et al., 2020). In the study, the improvement in the nutrient concentrations of safflower seeds may be due to more effective mobilizing mineral nutrients from the soil because of enhanced excretion of organic acids through rhizosphere bacteria (Glick, 1995; Biswas et al., 2000; Chandra et al., 2019). Another possible reason for the improved nutrients from the PGPR is the roots enhancement by producing phytohormones, resulting in a larger root surface, and therefore, has positive effects on plant acquisition of nutrients and water (Vardharajula et al., 2011; Wang et al., 2012). In addition, an increase in zinc uptake because of root-induced changes (i.e. enhanced root growth, surface area and activity) in the rhizosphere after the application of PGPR has also been reported in lettuce (Sahin et al., 2015). More recently, many studies have reported that the integrated use of humic acids and bacterial inoculation would be provide the higher nutrient uptake and plant performance in the various stress conditions, and ultimately result in vigorous, well-established and healthy plants (Esringu et al., 2016; Silva et al., 2017; Olivares et al., 2017). Similarly, Baldatto et al. (2010) and Schoebitz et al. (2016) also reported that inoculation of PGPR in combination with humic substances increased root and shoot biomass of pineapple and blueberry plants, and nutrient contents increased by $80 \% \mathrm{~K}, 131 \% \mathrm{P}$ and $132 \% \mathrm{~N}$ in pineapple, and 55\% $\mathrm{N}$ and $56 \% \mathrm{~K}$ in blueberry, compared to uninoculated control.

\section{Conclusions}

In conclusion, the current study comprehensively evaluated the effect of humic acids (200 and $400 \mathrm{~kg} \mathrm{ha}^{-1}$ ) and two promising Bacillus spp. strain on the growth, yield and quality of safflower under rainfed and irrigated field conditions. The study revealed that $400 \mathrm{~kg}$ humic acid ha ${ }^{-1}$ with the Bacillus subtilis OSU142 strain was the most effective combination for growth, seed and oil yields, and seed quality of safflower under irrigated conditions. Moreover, the integrated use of humic acids and PGPR alleviated the unfavorable effects of water deficit under rainfed conditions, and presented an improving strategy for the remediation of water stress in the safflower production in arid and semi-arid regions. The present results suggest that the combined use of humic acids and PGPR can be used as a biotechnological intervention for the alleviation of water deficit stress in safflower production in the arid and semiarid regions.

Acknowledgements. The author sincerely thanks for the valuable contributions to Prof. Dr. Ismail Hakki Ekin (bacteriological procedures), Dr. M. Figen Donmez (providing Bacillus spp.), Agriculture engineer Faruk Oguz (experiment and Lab supporting) and Prof. Dr. Abdullah Yesilova (data analyses).

\section{REFERENCES}

[1] Arkhipova, T. N., Prinsen, E., Veselov, S. U. et al. (2007): Cytokinin producing bacteria enhance plant growth in drying soil. - Plant Soil 292: 305-315. DOI: 10.1007/s11104007-9233-5.

[2] Arshad, M., Shaharoona, B., Mahmood, T. (2008): Inoculation with Pseudomonas spp. containing ACC-deaminase partially eliminates the effects of drought stress on growth, yield, and ripening of pea (Pisum sativum L.). - Pedosphere 18: 611-620. DOI: 10.1016/S1002-0160(08)60055-7. 
[3] Backer, R., Rokem, J. S., Ilangumaran, G., Lamont, J., Praslickova, D., Ricci, E., et al. (2018): Plant growth-promoting rhizobacteria: context, mechanisms of action, and roadmap to commercialization of biostimulants for sustainable agriculture. - Front. Plant Sci. 9: 1473. DOI: 10.3389/fpls.2018.01473.

[4] Baldotto, L. E. B., Baldotto, M. A., Canellas, L. P., Bressan-Smith, R., Olivares, F. L. (2010): Growth promotion of pineapple 'vitória' by humic acids and Burkholderia spp. during acclimatization. - Revista Bras. Cienc. Solo 34(5): 1593-1600. DOI: 10.1590/S0100-06832010000500012.

[5] Belimov, A. A., Dodd, I. C., Hontzeas, N., Theobald, J. C., Safronova, V. I., Davies, W. J. (2009): Rhizosphere bacteria containing 1- aminocyclopropane-1 -carboxylate deaminase increase yield of plants grown in drying soil via both local and systemic hormone signalling. - New Phytol. 181: 413-423.

[6] Biswas, J. C., Ladha, J. K., Dazzo, F. B., Yanni, Y. G., Rolfe, B. G. (2000): Rhizobia inoculation influences seedling vigor and yield of rice. - Agron J 92: 880-886. DOI: 10.2134/agronj2000.925880x.

[7] Bossio, D. A., Scow, K. M., Gunapala, N., Graham, K. J. (1998): Determinants of soil microbial communities: effects of agricultural management, season, and soil type on phospholipid fatty acid profiles. - Microb. Ecol. 36: 1-12. DOI: 10.1007/s002489900087.

[8] Busato, J. G., Silva, L. L., Aguiar, N. O., Canellas, L. P., Olivares, F. L. (2012): Changes in labile phosphorus forms during maturation of vermicompost enriched with phosphorus-solubilizing and diazotrophic bacteria. - Bioresour. Technol. 110: 390-395. DOI: 10.1016/j.biortech.2012.01.126.

[9] Cakmakci, R., Kantar, F., Sahin, F. (2001): Effect of N2-fixing bacterial inoculations on yield of sugar beet and barley. - J. Plant Nutr. Soil Sci. 164: 527-531. DOI: 10.1002/1522624(200110)164:5<527::AID-JPLN527>3.0.CO;2-1.

[10] Cakmakci, R., Dönmez, F., Aydin, A., Sahin, F. (2006): Growth promotion of plants by plant growth promoting rhizobacteria under greenhouse and two different field soil conditions. - Soil Biol. and Biochem. 38: 1482-1487. DOI: 10.1016/j.soilbio.2005.09.019.

[11] Canellas, L. P., Olivares, F. L. (2014): Physiological responses to humic substances as plant growth promoter. - Chem Biol Technol Agric. 1: 3. DOI: 10.1186/2196-5641-1-3.

[12] Canellas, L. P., Balmori, D. M., Médici, L. O., Aguiar, N. O., Campostrini, E., Rosa, R. C. C., et al. (2013): A combination of humic substances and Herbaspirillum seropedicae inoculation enhances the growth of maize (Zea mays L.). - Plant Soil 366: 119-132. DOI: 10.1007/s11104-012-1382-5.

[13] Cao, S., Zhou, X. R., Wood, C. C., Green, A. G., Singh, S. P., Liu, L., et al. (2013): A large and functionally diverse family of Fad2 genes in safflower (Carthamus tinctorius L.). - BMC Plant Biol. 13(1): 5. DOI: 10.1186/1471-2229-13-5.

[14] Cassán, F., Perrig, D., Sgroy, V., Masciarelli, O., Penna, C., Luna, V. (2009): Azospirillum brasilense Az39 and Bradyrhizobium japonicum E10 inoculated singly or in combination, promote seed germination and early seedling growth in corn (Zea mays L.) and soybean (Glycine max L.). - Eur J Soil Biol. 45: 28-35. DOI: 10.1016/j.ejsobi.2008.08.005.

[15] Chandra, D., Srivastava, R., Gupta, V. V. S. R., Franco, C. M. M., Paasricha, N., Saifi, S. K., et al. (2019): Field performance of bacterial inoculants to alleviate water stress effects in wheat (Triticum aestivum L.). - Plant Soil 441: 261-281. DOI: 10.1007/s11104-01904115-9.

[16] Chi, F., Shen, S. H., Cheng, H. P., Jing, Y. X., Yanni, Y. G., Dazzo, F. D. (2005): ascending migration of endophytic rhizobia, from roots to leaves, inside rice plants and assessment of benefits to rice growth physiology. - Applied and Environmental Microbiology 71(11): 7271-7278. DOI: 10.1128/AEM.71.11.7271-7278.2005. 
[17] Comas, L. H., Becker, V., Cruz, V. M. V., Byrne, P. F., Dierig, D. A. (2013): Root trials contributing to plant productivity under drought. - Frontiers in Plant Science 4: 442. DOI: $10.3389 / \mathrm{fpls} .2013 .00442$.

[18] Dajue, L., Mündel, H. H. (2006): Safflower. Carthamus tinctorius L. Promoting the Conservation and Use of Underutilized and Neglected Crops. - Institute of Plant Genetics and Crop Plant Research, Gatersleben/International Plant Genetic Resources Institute, Rome.

[19] Dobbss, L. B., Canellas, L. P., Olivares, F. L., Aguiar, N. O., Peres, L. E. P., Azevedo, M., et al. (2010): Bioactivity of chemically transformed humic matter from vermicompost on plant root growth. - J. Agric. Food Chem. 58: 3681-3688. DOI: 10.1021/jf904385c.

[20] Drenovsky, R. E., Vo, D., Graham, K. J., Scow, K. M. (2004): Soil water content and organic carbon availability are major determinants of soil microbial community composition. - Microb. Ecol. 48: 424-430. DOI: 10.1007/s00248-003-1063-2.

[21] Ekin, Z. (2005): Resurgence of safflower (Carthamus tinctorius L.) utilization: a global view. - Journal of Agronomy 4(2): 83-87. DOI: 10.3923/ja.2005.83.87.

[22] Ekin, Z. (2019): Integrated use of humic acid and plant growth promoting rhizobacteria to ensure higher potato productivity in sustainable agriculture. - Sustainability 11: 3417. DOI: $10.3390 /$ su11123417.

[23] El-Howeity, M. A., Asfour, M. M. (2012): Response of some varieties of canola plant (Brassica napus L.) cultivated in a newly reclaimed desert to plant growth promoting rhizobacteria and mineral nitrogen fertilizer. - Annals of Agricultural Sciences 57(2): 129-136. DOI: 10.1016/j.aoas.2012.08.006.

[24] Esitken, A., Karlidag, H., Ercisli, S., Turan, M., Sahin, F. (2003): The effect of spraying a growth promoting bacterium on the yield, growth and nutrient element composition of leaves of apricot (Prunus armeniaca L. cv. Hacihaliloglu). - Australian J. of Agric. Res. 54: 377-380. DOI: 10.1071/AR02098.

[25] Esringu, A., Kaynar, D., Turan, M., Ercisli, S. (2016): Ameliorative effect of humic acid and plant growth-promoting rhizobacteria (PGPR) on Hungarian vetch plants under salinity stress. - Commun Soil Sci Plant Anal. 47: 602-18. DOI: 10.1080/00103624.2016.1141922.

[26] Garcia-Pausas, J., Paterson, E. (2011): Microbial community abundance and structure are determinants of soil organic matter mineralisation in the presence of labile carbon. - Soil Biol. Biochem. 43: 1705-1713. DOI: 10.1016/j.soilbio.2011.04.016.

[27] Glick, B. R. (1995): The enhancement of plant growth by free-living bacteria. - Can J Microbiol 41: 109-117.

[28] Glick, B. R. (2014): Bacteria with ACC deaminase can promote plant growth and help to feed the world. - Microbiol Res. 169: 30-39. DOI: 10.1016/j.micres.2013.09.009.

[29] Gupta, S., Pandey, S. (2019): Unravelling the biochemistry and genetics of ACC deaminase - an enzyme alleviating the biotic and abiotic stress in plants. - Plant Gene. 18: 100175. DOI: 10.1016/j.plgene.2019.100175.

[30] Hoyos-Carvajal, L., Orduz, S., Bissett, J. (2009): Growth stimulation in bean (Phaseolus vulgaris L.) by Trichoderma. - Biol Control 51: 409-416. DOI: 10.1016/j.biocontrol.2009.07.018.

[31] Hungria, M., Campo, R. J., Souza, E. M., Pedrosa, F. O. (2010): Inoculation with selected strains of Azospirillum brasilense and A. lipoferum improves yields of maize and wheat in Brazil. - Plant Soil. 331: 413-425. DOI: 10.1007/s11104-009-0262-0.

[32] Kizil, S., Cakmak, Ö., Kirici, S., Inan, M. (2008): A comprehensive study on safflower (Carthamus tinctorius L.) in semi-arid conditions. - Biotechnol. Biotechnol. Equip. 22: 947-953. doi.org/10.1080/13102818.2008.10817585.

[33] Kristin, A., Miranda, H. (2013): The root microbiota - a fingerprint in the soil? - Plant Soil 370: 671-686. DOI: 10.1007/s11104-013-1647-7.

[34] Kumar, S., Ambreen, H., Variath, M. T., Rao, A. R., Agarwal, M., Kumar, A., et al. (2016): Utilization of molecular, phenotypic, and geographical diversity to develop 
compact composite core collection in the oilseed crop, safflower (Carthamus tinctorius L.) through maximization strategy. - Front. Plant Sci. 7: 1554. DOI: 10.3389/fpls.2016.01554.

[35] La Bella, S., Tuttolomond, T., Lazzeri, L., Matteo, R., Leto, C., Licata, M. (2019): An agronomic evaluation of new safflower (Carthamus tinctorius L.) germplasm for seed and oil yields under Mediterranean climate conditions. - Agronomy 9: 468. DOI: 10.3390/agronomy9080468.

[36] Lucas, J. A., Solano, B. R., Montes, F., Ojeda, J., Megias, M., Mañero, F. J. G. (2009): Use of two PGPR strains in the integrated management of blast disease in rice (Oryza sativa L.) in Southern Spain. - Field Crops Res. 114: 404-410. DOI: 10.1016/j.fcr.2009.09.013.

[37] Marulanda, A., Barea, J. M., Azcón, R. (2009): Stimulation of plant growth and drought tolerance by native microorganisms (AM Fungi and Bacteria) from dry environments: mechanisms related to bacterial effectiveness. - J Plant Growth Regul. 28: 115-124. DOI: 10.1007/s00344-009-9079-6.

[38] Mirzakhani, M., Ardakani, M. R., Aeene Band, A., Rejalı, F., Shirani Rad, A. H. (2009): Response of spring safflower to co-inoculation with Azotobacter chroococum and Glomus intraradices under different levels of nitrogen and phosphorus. - American J. of Agric. and Biol. Sci. 4: 255-261. DOI: 10.3844/ajabssp.2009.255.261.

[39] Mutumba, F. A., Zagal, E., Gerding, M., Castillo-Rosales, D., Schoebitz, M. (2018): Plant growth promoting rhizobacteria for improved water stress tolerance in wheat genotypes. - Journal of Soil Science and Plant Nutr. 18(4): 1080-1096. DOI: 10.4067/S0718-95162018005003003.

[40] Nardi, S., Carletti, P., Pizzeghello, D., Muscolo, A. (2009): Biological Activities of Humic Substances. - In: Seni, N., Xing, B., Huang, P. M. (eds.) Biophysico-Chemical Processes Involving Natural Nonliving Organic Matter in Environmental Systems. Wiley, New Jersey, pp. 305-340.

[41] Nelson, L. M. (2004): Plant growth promoting rhizobacteria (PGPR): prospects for new inoculants. - Crop Manag. 3: 301-305. DOI: 10.1094/cm-2004-0301-05-rv.

[42] Nosheen, A., Bano, A., Ullah, F., Farooq, U., Yasmin, Y., Hussain, I. (2011): Effect of plant growth promoting rhizobacteria on root morphology of Safflower (Carthamus tinctorius L.). - African J. of Biotech. 10(59): 12639-12649. DOI: 10.5897/AJB11.1647.

[43] Nosheen, A., Bano, A., Yasmin, H., Keyani, R., Habib, R., Shah, S. T. A., et al. (2016): Protein quantity and quality of safflower seed improved by NP fertilizer and Rhizobacteria (Azospirillum and Azotobacter spp.). - Front. Plant Sci. 7: 104. DOI: 10.3389/fpls.2016.00104.

[44] Nosheen, A., Naz, R., Tahir, A. T., Yasmin, H., Keyani, R., Mitrevski, B., Bano, A., Chin, S. T., Marriott, P. J. (2018): Improvement of safflower oil quality for biodiesel production by integrated application of PGPR under reduced amount of NP fertilizers. PLoS ONE 13: e0201738. https://doi.org/10.1371/journal.

[45] Olivares, F. L., Aguiar, N. O., Rosa, R. C. C., Canellas, L. P. (2015): Substrate biofortification in combination with foliar sprays of plant growth promoting bacteria and humic substances boosts production of organic tomatoes. - Sci. Hortic. 183: 100-108. DOI: 10.1016/j.scienta.2014.11.012.

[46] Olivares, F. L., Busato, J. G., de Paula, A. M., Lima, L. S., Aguiar, N. O., Canellas, L. P. (2017): Plant growth promoting bacteria and humic substances: crop promotion and mechanisms of action. - Chem. Biol. Technol. Agric. 4: 30. DOI: 10.1186/s40538-0170112-x.

[47] Orhan, E., Esitken, A., Ercisli, S., Turan, M., Fikrettin, S. (2006): Effects of plant growth promoting rhizobacteria (PGPR) on yield, growth and nutrient contents in organically growing raspberry. - Sci. Hort. 111: 38-43. DOI: 10.1016/j.scienta.2006.09.002. 
[48] Ozturk, E., Ozer, H., Polat, T. (2008): Growth and yield of safflower genotypes grown under irrigated and non-irrigated conditions in a highland environment. - Plant Soil Environ. 54(10): 453-460. DOI: 10.17221/403-PSE.

[49] Puglisi, E., Pascazio, S., Suciu, N., Cattani, I., Fait, G., Spaccini, R., et al. (2013): Rhizosphere microbial diversity as influenced by humic substance amendments and chemical composition of rhizodeposits. - J. Geochem. Explor. 129: 82-94. DOI: 10.1016/j.gexplo.2012.10.006.

[50] Rana, A., Joshi, M., Prasanna, R., Shivay, Y. S., Nain, L. (2012): Biofortification of wheat through inoculation of plant growth promoting rhizobacteria and cyanobacteria. European J Soil Biol. 50: 118-26. DOI: 10.1016/j.ejsobi.2012.01.005.

[51] Rapson, S., Wu, M., Okada, S., Das, A., Shrestha, P., Zhou, X. R., et al. (2015): A case study on the genetic origin of the high oleic acid trait through FAD2-1DNA sequence variation in safflower (Carthamus tinctorius L.). - Frontiers in Plant Science 6: 691. DOI: 10.3389/fpls.2015.00691.

[52] Rong, Q., Zhong, K., Huang, H., Li, C., Zhang, C., Nong, X. (2020): Humic acid reduces the available cadmium, copper, lead, and zinc in soil and their uptake by tobacco. - Appl. Sci. 10: 1077. DOI: 10.3390/app10031077.

[53] Rosas, S. B., Avanzin, G., Carlier, E., Pasluosta, C., Pastor, N., Rovera, M. (2009): Root colonization and growth promotion of wheat and maize by Pseudomonas aurantiaca SR1. - Soil Biol Biochem. 41: 1802-1806. DOI: 10.1016/j.soilbio.2008.10.009.

[54] Rubin, R. L., van Groenigen, K. J., Hungate, B. A. (2017): Plant growth promoting rhizobacteria are more effective under drought: a meta-analysis. - Plant and Soil 1-15. DOI: $10.1007 / \mathrm{s} 11104-017-3199-8$.

[55] Sahin, U., Ekinci, M., Kiziloglu, F. M., Yildirim, E., Turan, M., et al. (2015): Ameliorative effects of plant growth promoting bacteria on water-yield relationships, growth, and nutrient uptake of lettuce plants under different irrigation levels. HortScience 50(9): 1379-1386. DOI: 10.21273/HORTSCI.50.9.1379.

[56] Sandhya, V., Ali, S. Z., Grover, M. et al. (2010): Effect of plant growth promoting Pseudomonas spp. on compatible solutes, antioxidant status and plant growth of maize under drought stress. - Plant Growth Regul. 62: 21-30. DOI: 10.1007/s10725-010-94794.

[57] Schmidt, W., Santi, S., Pinton, R., Varanini, Z. (2007): Water-extractable humic substances alter root development and epidermal cell pattern in Arabidopsis. - Plant Soil 300: 259-267. DOI: 10.1007/s11104-007-9411-5.

[58] Schoebitz, M., López, M. D., Serrí, H., Martínez, O., Zagal, E. (2016): Combined application of microbial consortium and humic substances to improve the growth performance of blueberry seedlings. - J Soil Sci Plant Nutr. 16: 1010-23. DOI: 10.4067/S0718-95162016005000074.

[59] Shahid, M., Hameed, S., Imran, A., Ali, S., Elsas, J. D. (2012): Root colonization and growth promotion of sunflower (Helianthus annuus L.) by phosphate solubilizing Enterobacter sp. Fs-11. - World J Microbiol Biotechnol. 28: 2749-2758. DOI: 10.1007/s11274-012-1086-2.

[60] Sharifi, R. S., Namvar, A., Sharifi, R. S. (2017): Grain filling and fatty acid composition of safflower fertilized with integrated nitrogen fertilizer and biofertilizers. - Pesq. Agropec. Bras., Brasília 52(4): 236-243. DOI: 10.1590/s0100-204x2017000400003.

[61] Silva, S. F., Olivares, F. L., Canellas, L. P. (2017): The biostimulant manufactured using diazotrophic endophytic bacteria and humates is effective to increase sugarcane yield. Chem Biol Technol Agric. 4: 24. DOI: 10.1186/s40538-017-0106-8.

[62] Soleymanifard, A., Sidat, S. A. (2011): Effect of inoculation with bio-fertilizer in different nitrogen levels on yield and yields components of safflower under dry land conditions. - American Eurasian J. of Agric. and Envir. Sci. 11: 473-477. https://www.idosi.org/aejaes/jaes11(4)11/3.pdf. 
[63] Somerville, C., Briscoe, J. (2001): Genetic engineering and water. - Science 292(5525): 2217. DOI: $10.1126 /$ science.292.5525.2217.

[64] Thonar, C., Lekfeldt, J. D. S., Cozzolino, V., Kundel, D., Kulhánek, M., Mosimann, C., et al. (2017): Potential of three microbial bio-effectors to promote maize growth and nutrient acquisition from alternative phosphorous fertilizers in contrasting soils. - Chem Biol Technol Agric. 4: 7. DOI: 10.1186/s40538-017-0088-6.

[65] Vardharajula, S., Zulfikar, A. S., Grover, M., Reddy, G., Bandi, V. (2011): Droughttolerant plant growth promoting Bacillus spp.: effect on growth, osmolytes, and antioxidant status of maize under drought stress. - J Plant Interact. 6: 1-14. DOI: 10.1080/17429145.2010.535178.

[66] Vejan, P., Abdullah, R., Khadiran, T., Ismail, S., Nasrulhaq Boyce, A. (2016): Role of plant growth promoting rhizobacteria in agricultural sustainability-a review. Molecules 21: 573. DOI: 10.3390/molecules21050573.

[67] Vivas, A., Marulanda, A., Ruiz-Lozano, J. M., et al. (2003): Influence of a Bacillus sp. on physiological activities of two arbuscular mycorrhizal fungi and on plant responses to PEG-induced drought stress. - Mycorrhiza 13: 249-256. DOI: 10.1007/s00572-003-0223$\mathrm{z}$.

[68] Wang, C. J., Yang, W., Wang, C., Gu, C., Niu, D. D., Liu, H. X., et al. (2012): Induction of drought tolerance in cucumber plants by a consortium of three plant growth-promoting rhizobacterium strains. - PLoS One 7: e52565. DOI: 10.1371/journal.pone.0052565.

[69] Weiss, E. A. (1983): Safflower. - In: Weiss, E. A. (ed.) Oilseed Crops. Longman, London, pp. 216-281. 